\title{
Neuroinflammatory processes are augmented in mice overexpressing human heat-shock protein B1 following ethanol- induced brain injury
}

Brigitta Dukay ${ }^{1,2^{*}{ }^{4}}$, Fruzsina R. Walter ${ }^{3}$, Judit P. Vigh ${ }^{3}$, Beáta Barabási ${ }^{3,4}$, Petra Hajdu ${ }^{1}$, Tamás Balassa ${ }^{1,5}$, Ede Migh ${ }^{1}$, András Kincses ${ }^{3}$, Zsófia Hoyk ${ }^{3}$, Titanilla Szögi ${ }^{6}$, Emőke Borbély ${ }^{6}$, Bálint Csoboz ${ }^{1,7}$, Péter Horváth ${ }^{1,8}$, Lívia Fülöp ${ }^{6}$, Botond Penke ${ }^{6}$, László Vígh ${ }^{1}$, Mária A. Deli ${ }^{3}$, Miklós Sántha ${ }^{1 \dagger}$ and Melinda E. Tóth ${ }^{{ }^{*}+}$

\section{Abstract}

Background: Heat-shock protein B1 (HSPB1) is among the most well-known and versatile member of the evolutionarily conserved family of small heat-shock proteins. It has been implicated to serve a neuroprotective role against various neurological disorders via its modulatory activity on inflammation, yet its exact role in neuroinflammation is poorly understood. In order to shed light on the exact mechanism of inflammation modulation by HSPB1, we investigated the effect of HSPB1 on neuroinflammatory processes in an in vivo and in vitro model of acute brain injury.

Methods: In this study, we used a transgenic mouse strain overexpressing the human HSPB1 protein. In the in vivo experiments, 7-day-old transgenic and wild-type mice were treated with ethanol. Apoptotic cells were detected using TUNEL assay. The mRNA and protein levels of cytokines and glial cell markers were examined using RT-PCR and immunohistochemistry in the brain. We also established primary neuronal, astrocyte, and microglial cultures which were subjected to cytokine and ethanol treatments. TNFa and hHSPB1 levels were measured from the supernates by ELISA, and intracellular hHSPB1 expression was analyzed using fluorescent immunohistochemistry.

Results: Following ethanol treatment, the brains of hHSPB1-overexpressing mice showed a significantly higher mRNA level of pro-inflammatory cytokines (Tnf, II1 b), microglia (Cd68, Arg1), and astrocyte (Gfap) markers compared to wild-type brains. Microglial activation, and 1 week later, reactive astrogliosis was higher in certain brain areas of ethanol-treated transgenic mice compared to those of wild-types. Despite the remarkably high expression of pro-apoptotic Tnf, hHSPB1-overexpressing mice did not exhibit higher level of apoptosis. Our data suggest that intracellular hHSPB1, showing the highest level in primary astrocytes, was responsible for the inflammation-regulating effects. Microglia cells were the main source of TNFa in our model. Microglia isolated from hHSPB1-overexpressing mice showed a significantly higher release of TNFa compared to wild-type cells under inflammatory conditions.

(Continued on next page)

\footnotetext{
*Correspondence: dukay.brigitta@brc.hu; toth.erzsebetmelinda@brc.hu Miklós Sántha and Melinda E. Tóth are shared last authors.

${ }^{1}$ Institute of Biochemistry, Biological Research Centre, Temesvári krt. 62, Szeged H-6726, Hungary

Full list of author information is available at the end of the article
} 
(Continued from previous page)

Conclusions: Our work provides novel in vivo evidence that hHSPB1 overexpression has a regulating effect on acute neuroinflammation by intensifying the expression of pro-inflammatory cytokines and enhancing glial cell activation, but not increasing neuronal apoptosis. These results suggest that hHSPB1 may play a complex role in the modulation of the ethanolinduced neuroinflammatory response.

Keywords: hHSPB1, Heat-shock protein, Astroglia, Cytokines, Ethanol toxicity, Microglia, Neuron, Neuroinflammation, Transgenic mice, Primary cells

\section{Background}

Neuroinflammation plays a central role in the pathophysiology of most neurological diseases. The activation of astro- and microglia cells, and their subsequent production of cytokines and chemokines are the main characteristics of neuroinflammation. Inflammation in the brain is primarily associated with the process of neurodegeneration, as it is initiated in order to aid the repair of the damaged brain area. However, in the case if it becomes dysregulated, a chronic inflammation could appear which further aggravates tissue damage. Therefore, the tight regulation of the neuroinflammatory response during neuronal tissue damage is crucial in preventing or at least in reducing the negative effects of chronic inflammation [1]. Heat-shock proteins (HSPs) could be one of the potential candidates for such an inflammation-regulating role, as they show a rapid induction under stress conditions, they promote cell survival under these conditions, and their modulatory effects on inflammatory processes are well described [2].

Heat-shock protein B1 (HSPB1, HSP27/25) is one of the most well-studied members of the evolutionarily conserved small heat-shock protein family. These ATPindependent chaperon proteins are characterized by low molecular weight between 16 and $40 \mathrm{kDa}$, and a conserved $\mathrm{C}$-terminal $\alpha$-crystallin domain. As molecular chaperones, small heat-shock proteins have an important role in maintaining the normal cellular protein homeostasis by binding to partially denatured proteins, thereby preventing their irreversible aggregation [3]. In the brain, HSPB1 is the most abundant in astrocytes under physiological conditions and it is even more elevated in response to various stress conditions, such as increased temperature or oxidative stress $[4,5]$. Increased astroglial expression of HSPB1 was also detected after transient focal ischemia and in neurodegenerative disorders associated with pathological protein inclusions, such as tauopathies or Alzheimer's disease [4, 6-8]. This line of observations suggests that HSPB1 might also play a role in reactive astrogliosis. Compared to astrocytes, HSPB1 is present in neurons to a lesser extent, although it was also described to be upregulated in pathological and under stress conditions $[4,9,10]$. In microglia, HSPB1 is present only in negligible amounts but following hyperthermia, it showed an elevated expression [11].

Besides its well-described role as a chaperon, HSPB1 is also associated with neuroinflammation through several mechanisms. HSPB1 can help to maintain the integrity of cytoskeletal networks through its association with various cytoskeletal filaments, such as the glial fibrillary acidic protein (GFAP), vimentin (Vim), or neurofilament $[12,13]$. HSPB1 can also interact with several components of the apoptotic pathways and exert a strong antiapoptotic function [14]. Moreover, it can modulate the release of cytokines from various cells; however, a multitude of effects can be observed in different studies in that regard. There has been an observation that described an HSPB1-dependent interleukin-10 (IL-10) increase and an associated anti-inflammatory response [15]. However, another study pointed towards a more pro-inflammatory role for HSPB1 as it was observed to induce the production of IL- 8 and reduce the expression of anti-inflammatory transforming growth factor- $\beta 1$ (TGF- $\beta 1$ ) and the cluster of differentiation 40 (CD40) ligand in astrocytes in vitro [16]. In addition, HSPB1 is described to regulate the activation of the nuclear factor kappa B (NFkB) pathway. Recently, intracellular HSPB1 has been demonstrated to promote the degradation of IkappaB kinase- $\beta$ resulting in an inactive state of NFkB and a subsequent decrease in tumor necrosis factor- $\alpha$ (TNF $\alpha)$ production in microglial cells [17]. However, in a macrophage culture, extracellular HSPB1 treatment was observed to activate the NFkB pathway via the degradation of the inhibitor of nuclear factor-kappa B- $\alpha$ $(\mathrm{I} \kappa \mathrm{B} \alpha)$, thereby upregulating both pro- and antiinflammatory factors, such as IL1b, TNF, IL-10, and the granulocyte-macrophage colony-stimulating factor [18]. Moreover, HSPB1 expressed in endothelial cells of the brain microvasculature contributes to an indirect protection against neuroinflammation. HSPB1 was described to ameliorate the impairment of the blood-brain barrier, and by this decreasing the influx of peripheral immune cells into the brain parenchyma after ischemic injury [19]. Overall, a growing number of evidence indicates that HSPB1 can affect the inflammation-related processes in several ways, but it seems to induce diverse effects depending on the cell type and the extra- or 
intracellular presence of the protein. Despite the diversity of the observed actions for HSPB1 in the central nervous system, the inflammation modulatory effects of this protein would require further clarification. In addition, there is a lack of comprehensive in vivo studies investigating the impact of HSPB1 on multiple components of inflammation within the CNS.

In this study, we aimed to test the hypothesis whether HSPB1 is involved in the regulation of cytokine expression, gliosis, and apoptosis in ethanol-induced acute neuroinflammation. We analyzed the inflammation-related processes using our previously generated human HSPB1 (hHSPB1)-overexpressing transgenic mouse strain [20], as well as using primary cell cultures isolated from the transgenic mouse model. Ethanol treatment is a suitable method for modeling the sterile inflammatory environment that serves as a central disease-promoting mechanism in many neurological disorders. In our in vivo experiments, we used early postnatal acute ethanol exposure because, due to the particularly high sensitivity of the developing brain to alcohol toxicity, even a single ethanol treatment leads to robust neurodegeneration, which is accompanied by neuroinflammatory processes, including cytokine release and glial cell activation [21, 22]. Accordingly, with this method, we were able to induce neuroinflammation rapidly in the brains of 7-day-old hHSPB1overexpressing transgenic and wild-type mice and to investigate the possible immunomodulatory effects of hHSPB1 overexpression in this sterile inflammatory milieu. hHSPB1-overexpressing mice showed enhanced expression of pro-inflammatory cytokines $T n f$ and $I l 1 b$ as well as increased immunoreactivity of astrocyte and microglial activation markers in response to ethanolinduced acute brain injury, whereas they exhibited a nonsignificant trend of decreased apoptosis. In addition, in vitro results showed that the release of hHSPB1 from the cells is minimal even after stress treatment. Therefore, we suggest that the intracellular form of hHSPB1 is responsible for the observed inflammation-regulating effects, showing a significantly increased expression in primary astrocytes after cytokine and ethanol treatment. Moreover, microglia seemed to be the main source of pro-inflammatory cytokines in vitro, since the highest TNF $\alpha$ response to cytokine treatment was detected in the supernates from microglia cultures from hHSPB1 transgenic animals. Taken together, we found that the ethanol-induced neuroinflammatory processes were aggravated in our HSPB1-overexpressing mice including the pro-inflammatory cytokine expression and glial cell activation, while we could not detect the further increase of the inflammation-related neuronal damage in these animals, suggesting that HSPB1 has a complex role in the regulation of ethanol-induced acute inflammation.

\section{Methods \\ In vivo experiments \\ Animals}

The study has been carried out according to the EU Directive 2010/63/EU and was approved by the regional National Food Chain Safety Agency and Animal Health Directorate (Csongrad-county, Hungary) under the project license XVI/4136/2014. Mice were housed in groups of two to three under standard conditions $\left(24^{\circ} \mathrm{C}, 12 \mathrm{~h}\right.$ of light-dark cycle) with food and water available ad libitum. Seven-day-old hHSPB1 transgenic mice and wildtype littermates were used for the studies. The hHSPB1overexpressing transgenic line was previously established by our group on a homogenous C57BL/6 genetic background [20].

\section{Ethanol treatment}

Neurodegeneration was induced in 7-day-old wild-type and hHSPB1-overexpressing transgenic mice by the subcutaneous injection of $20 \%$ ethanol solution as described earlier in Ikonomidou et al. [21]. Ethanol was administered in two equal doses of $2.5 \mathrm{~g} / \mathrm{kg}$ body weight within 2 $\mathrm{h}$ (at zero and at the 2nd hour), resulting in a total dose of $5 \mathrm{~g} / \mathrm{kg}$. Control pups were treated with physiological saline. After cervical dislocation and decapitation, brains were removed at $7 \mathrm{~h} / 24 \mathrm{~h} / 1$ week after the second injection and processed as follows: the dissected brains were embedded in optimal cutting temperature (OCT) compound (Tissue-Tek, Sakura Finetek, CA, USA) for cryostat sectioning and to carry out the terminal deoxynucleotidyl transferase dUTP nick end labeling (TUNEL) assay; for immunohistochemistry, brains were immersion-fixed in $3 \%$ paraformaldehyde (PFA)- $0.1 \mathrm{M}$ phosphate buffer solution; the brains were further homogenized in AccuZol Reagent for RNA isolation or in radioimmunoprecipitation assay (RIPA) buffer for protein isolation. At the same time, small tail biopsies were taken for subsequent genomic DNA isolation and genotype determination.

\section{hHSPB1 Western blot analysis}

Protein level of transgenic human HSPB1 was determined from whole-brain homogenates of 7-day-old mice ( $n=3$ mice per group). Brains were homogenized in 1 $\mathrm{ml}$ RIPA buffer containing $2 \mathrm{mM}$ phenylmethylsulfonyl fluoride and $2 \mathrm{mMN}$-ethylmaleimide. After centrifugation $\left(13,000 \mathrm{~g}\right.$ for $25 \mathrm{~min}$ at $\left.4{ }^{\circ} \mathrm{C}\right)$, protein concentrations were measured in the supernates at $280 \mathrm{~nm}$ using a NanoDropND-1000 spectrophotometer (Nanodrop, DE, USA). Five milligram per milliliter samples were mixed with sample buffer containing sodium dodecyl sulfate (SDS), glycerol, $\beta$-mercaptoethanol, and bromophenol blue, and incubated at $95^{\circ} \mathrm{C}$ for $5 \mathrm{~min}$. Protein samples $(50 \mu \mathrm{g})$ were loaded into a $15 \%$ SDS-polyacrylamide gel and samples were run at $80 \mathrm{~V}$ for $2 \mathrm{~h}$ in $1 \times$ SDS-PAGE 
running buffer. Then, proteins were transferred to polyvinylidene difluoride membranes (Pall Corporation, NY, USA), were submerged in $1 \times$ transfer buffer, and run at $200 \mathrm{~mA}$ for $1 \mathrm{~h}$ on ice. Subsequently, membranes were incubated in phosphate-buffered saline with Tween 20 (PBST) containing 5\% nonfat milk powder for $1 \mathrm{~h}$ at room temperature. The following primary antibodies were used in the study: rabbit anti-hHSPB1 $(\mathrm{O} / \mathrm{N}$ at $4{ }^{\circ} \mathrm{C}$, Stressgen - Enzo Life Sciences, NY, USA, no crossreactivity with mouse HSPB1) and rabbit anti-actin (2 h at room temperature, Sigma-Aldrich Ltd, Budapest, Hungary). The secondary antibody used was horseradish peroxidase-conjugated goat anti-rabbit (Jackson ImmunoResearch Europe Ltd., Cambridgeshire, UK) (see Table S1 for details of antibodies). Afterwards, membranes were incubated with a chemiluminescent detection reagent (Luminata Forte Western HRP Substrate, Merck Millipore, MA, USA) for 2 min and blots were developed manually on X-ray films. Quantification of the results was performed using the open-access ImageJ software.

\section{$m R N A$ isolation and reverse transcription}

mRNA was isolated from whole brains of the mice. Homogenized tissue was mixed with AccuZol Reagent (Bioneer, South Korea), by adding $1 \mathrm{ml}$ to each tissue sample. Subsequently, $200 \mu \mathrm{l}$ chloroform was added to the samples followed by incubation on ice for $5 \mathrm{~min}$. Phases were separated by centrifugation at $13,000 \mathrm{~g}$ for $15 \mathrm{~min}$ at $4{ }^{\circ} \mathrm{C}$, and the aqueous phase was separated from the organic phase from where the RNA was precipitated with $100 \%$ isopropyl alcohol during incubation for $10 \mathrm{~min}$ at $-20^{\circ} \mathrm{C}$. After centrifugation for $10 \mathrm{~min}$ at 13 , $000 \mathrm{~g}$ at $4{ }^{\circ} \mathrm{C}$, the RNA pellets were washed with $80 \%$ ethanol and the samples were centrifuged for $5 \mathrm{~min}$ at $13,000 \mathrm{~g}$ at $4{ }^{\circ} \mathrm{C}$. The RNA pellets were dissolved in RNase-free water and cleaned by using Nucleo Spin mRNA Clean-Up columns (Macherey-Nagel, Düren, Germany). Samples were treated with DNase and were eluted from the membrane with RNase-free water. Concentrations of the samples were measured at $230 \mathrm{~nm}$ using a spectrophotometer (NanoDrop ND-1000, Nanodrop, DE, USA). mRNA samples were converted to cDNA using reverse transcription (High Capacity cDNA Reverse Transcription Kit, Applied Biosystems, CA, USA) according to the manufacturer's instructions.

\section{Real-time PCR analysis}

Real-time (RT)-PCR was performed to analyze the level of hHSPB1, cytokines, and glial markers. Gene-specific primers are listed in Table S2. Each reaction was performed in a total volume of $20 \mu \mathrm{l}$ containing $10 \mu \mathrm{l}$ of Power SYBR Green PCR Master Mix (Applied Biosystems, CA, USA), $1 \mu \mathrm{l}$ of $5 \mathrm{pmol} / \mu \mathrm{l}$ primer mix (forward + reverse), and $9 \mu \mathrm{l}$ of cDNA sample. The amplification was carried out on a RotorGene 3000 instrument (Corbett Research, Australia) with the following cycling parameters: heat activation at $95^{\circ} \mathrm{C}$ for $10 \mathrm{~min}$; followed by 45 cycles of denaturation at $95^{\circ} \mathrm{C}$ for $15 \mathrm{~s}$, annealing at $56^{\circ} \mathrm{C}$ for $15 \mathrm{~s}$, and extension at $60^{\circ} \mathrm{C}$ for $40 \mathrm{~s}$. Fluorescent signals were collected after each extension step at $72{ }^{\circ} \mathrm{C}$ and at the end, the registration of the melting curve was performed between 50 and $95^{\circ} \mathrm{C}$. Expression level of the target genes was normalized to an endogenous control (glyceraldehyde 3-phosphate dehydrogenase, Gapdh). Relative expression of the target genes compared to the untreated wild-type animals was calculated using the $\Delta \Delta \mathrm{Ct}$ method ( $n=8$ mice per group at $24 \mathrm{~h}$; $n=3$ mice per group at $7 \mathrm{~h}$ and 1 week).

\section{TUNEL assay}

To assess the level of apoptotic cell death, TUNEL assay was used, which labels the fragmented DNA. Brains were embedded in OCT compound, then $10-\mu \mathrm{m}$ sagittal frozen sections were prepared. Sections were post-fixed in $4 \%$ paraformaldehyde in phosphate-buffered saline (PBS) solution $(\mathrm{pH}=7.4)$ for $20 \mathrm{~min}$. Following a $30-\mathrm{min}$ PBS rinse, sections were blocked in $3 \% \mathrm{H}_{2} \mathrm{O}_{2}$ in methanol for $10 \mathrm{~min}$, then they were permeabilized with a solution of $0.1 \%$ Triton X-100 and incubated in $0.1 \%$ sodium citrate for $2 \mathrm{~min}$. After washing with PBS, $50 \mu \mathrm{l}$ of TUNEL reaction mixture was added to each sample (In situ Cell Death Detection Kit, POD, Roche Applied Science, IN, USA) and the sections were incubated for 1 $\mathrm{h}$ at $37^{\circ} \mathrm{C}$ in a humidified atmosphere. Under the same circumstances, the sections were incubated with Converter-POD solution for $30 \mathrm{~min}$, then washed in PBS, followed by a 10-min signal conversion with a peroxidase reagent. The red apoptotic cells were detected under a light microscope. For quantitative comparison, the TUNEL-positive cells were counted in fifteen fields of view of each brain section ( $n=6$ mice per group).

\section{Fluorescent immunohistochemistry}

Double immunostaining was performed to study the expression pattern of hHSPB1. After washing in PBS, 30$\mu \mathrm{m}$-thick frozen sagittal brain sections were permeabilized and blocked with $0.2 \%$ Triton X-100 and 3\% bovine serum albumin (BSA) in PBS for $1 \mathrm{~h}$ at room temperature. Then the sections were incubated overnight at $4{ }^{\circ} \mathrm{C}$ with the following primary antibodies: goat anti-IBA1 (ionized calcium-binding adaptor molecule 1, Abcam, Cambridge, UK), mouse anti-GFAP (Sigma-Aldrich Ltd, Budapest, Hungary), mouse anti-NEUN (neuronal nuclei, Merck Millipore, MA, USA), and rabbit anti-hHSPB1. Appropriate secondary antibodies were applied for $2 \mathrm{~h}$ : Alexa Fluor-488-conjugated rabbit anti-goat (Jackson ImmunoResearch Europe Ltd., Cambridgeshire, UK); FITCconjugated goat anti-mouse (Sigma-Aldrich Ltd, Budapest, 
Hungary); Alexa Fluor-647-conjugated goat anti-rabbit (Thermo Fisher Scientific, MA, USA) (Table S1). Cell nuclei were counterstained with 4',6-diamidino-2-phenylindole (DAPI) (Sigma-Aldrich Ltd, Budapest, Hungary), at the concentration of $0.5 \mu \mathrm{g} / \mathrm{ml}$ for $5 \mathrm{~min}$. Immunostainings were examined with a confocal laser scanning microscope (Olympus Fluoview FV1000, Olympus Life Science Europa GmbH, Hamburg, Germany).

\section{Peroxidase immunohistochemistry}

To analyze the activation of glial cells, brain sections were labeled with microglia and astrocyte markers. The brain region-specific expression pattern of the transgenic hHSPB1 protein was also examined with this method. First, the brain sections were treated with $20 \%$ methanol and $3 \% \mathrm{H}_{2} \mathrm{O}_{2}$ for 15 min to deactivate endogenous peroxidases. The sections were washed in PBS then blocked for $2 \mathrm{~h}$ at room temperature using $2 \%$ normal rabbit or horse serum and $0.3 \%$ BSA in PBS. Then the sections were incubated with rabbit anti-hHSPB1, mouse anti-GFAP, or goat anti-IBA1 primary antibodies overnight at $4{ }^{\circ} \mathrm{C}$. Subsequently, sections were incubated with the appropriate secondary antibodies: peroxidase-labeled goat anti-rabbit, peroxidase-conjugated rabbit anti-mouse (ChemiconMerck Millipore, MA, USA), or biotinylated donkey antigoat (Jackson ImmunoResearch Europe Ltd., Cambridgeshire, UK) for $2 \mathrm{~h}$ (Table S1). The sections were then incubated with the chromogen substrate, 3,3'-diaminobenzidine (DAB) (Sigma-Aldrich Ltd, Budapest, Hungary) at the concentration of $10 \mathrm{mg} / \mathrm{ml}$ for $15 \mathrm{~min}$, and the solution was supplemented with $1 \%$ nickel chloride. For IBA1 staining, an avidin-biotin (VECTASTAIN Elite ABC Peroxidase Kit, Vector Laboratories, CA, USA) treatment was applied for $2 \mathrm{~h}$ at room temperature as a signal amplification step before visualizing the immunoreaction with DAB. The immunostained sections were digitally scanned using a slide scanner (Mirax Midi, 3DHistech Ltd., Budapest, Hungary) ( $n=3$ mice per group and 3 sections per animal). Images were analyzed with the Pannoramic Viewer 1.15.4; CaseViewer 2.1 and QuantCenter; and HistoQuant module softwares (3DHistech Ltd., Budapest, Hungary). Each region of interest was manually outlined, then followed by automated detection of relative areas of immunopositivity. Results are given in percentage of the immunopositive areas compared to the outlined areas (relative area).

\section{Automated analysis of microglial morphology with deep learning approach}

Morphological categorization The activity state of microglia can be well characterized by their morphology, based on which microglia were classified into three subtypes: ramified (first class), intermediate (second class), and amoeboid (third class) [23, 24]. The ramified morphology of resting microglia is characterized by a small cell body and branching, long, thin processes extending far from the cell body (Fig. S1a). Cells classified into intermediate and amoeboid groups can be considered activated. The former is characterized by having an enlarged cell body and numerous thick, shortened processes which are still branching, while the latter is characterized by a large cell body which is completely round or has a few very short and thick primary processes (Fig. S1b).

Annotation procedure Manual annotations were performed using the AnnotatorJ software [25]. In total, 5883 cells on 232 images were annotated independently by two experts. Each annotated object in the set contains an associated class label and a bounding rectangle that was drawn around it. The total number of classes is 3 . The number of the annotated cells in the first class was 2961 and 1461-1461 in the second and third. Rectangle size can vary and they can intersect each other.

For performance evaluation, 30 images with a total of $\sim 800$ cells were selected. This validation set was annotated by both of the experts. Performing this annotation at two different time points, we measured the intraexpert accuracies.

Evaluation with deep learning Training the model: pytorch [26], a Python-based deep learning package was used to detect microglia (we used Python 3.6). We trained multiple models based on two different architectures, namely the yolov3-SPP [27] and CSPResNext50PANet-SPP [28]. To make the training robust, the training data was augmented using random affine transformations. We were using ADAM (Adaptive Moment Estimation [29]) optimizer with a base learning rate of 0.001 . Because for such data there are no pretrained weights available, we used random initialization. The number of epochs for each setup was 2000. This resulted in 0.594 as the best mAP (mean average precision) for yolov3-SPP and 0.593 as the best mAP for CSPResNext50-PANet-SPP. The training was performed on a GPU cluster with a $2.10 \mathrm{GHz} \operatorname{Intel}(\mathrm{R}) \mathrm{Xeon}(\mathrm{R}) \mathrm{ES}-2620$ CPU, 32 GB memory, and NVIDIA Titan Xp graphics card and for both cases took roughly $72 \mathrm{~h}$.

Microglia detection with classification We selected the yolov3-SPP model for the evaluation part because it resulted both statistically and visually in better results. To evaluate the performance of the proposed deep learning model, we measured precision, recall, and F1 score of two different annotators. The object matching between the detection and classification results and the ground truth was done manually by an expert. We 
considered a bounding box detection to be correct (true positive, TP) when it fully contained a cell; otherwise, it was considered as false positive (FP). When the detection algorithm was not able to find an object that was listed in the ground truth, that sample was treated as false negative (FN). Based on these conditions, we calculated the precision $(\mathrm{P})=\mathrm{TP} /(\mathrm{TP}+\mathrm{FP})$; recall $(\mathrm{R})=\mathrm{TP} /$ $(\mathrm{TP}+\mathrm{FN}) ; \mathrm{F} 1$ score $(\mathrm{F} 1)=2 * \mathrm{P} * \mathrm{R} /(\mathrm{P}+\mathrm{R})$; and detection accuracy $(\mathrm{DA})=\mathrm{TP} /(\mathrm{TP}+\mathrm{FP}+\mathrm{FN})$.

To inspect the accuracy of the classification part, we have checked all the images selected for the evaluation. As the detection accuracy was already calculated, we supervised the predicted class labels for each object. Based on the correctly $(\mathrm{C})$ and incorrectly (IC) predicted class labels, we calculated the classification accuracy $(\mathrm{CA})=$ (C) / (C + IC) (Fig. S1c).

For the final detection result, we evaluated four different comparisons. First, we measured self-accuracy of the human experts. This involves intra- and interexpert accuracies. We measured intraexpert accuracy by multiple annotations of the same images made by the same expert. We did this by annotating the same images with 2 weeks time difference. The intraexpert accuracies resulted in $97.4 \%$ accuracy $\quad$ (precision $=0.985$, recall $=0.989, \quad$ F1 score $=0.987$ ) and $97.8 \%$ accuracy (precision $=0.984$, recall $=0.993$, F1 score $=0.988$ ). Meanwhile, interexpert accuracy is the comparison of the annotations made by two experts on the same test set. This observation has reached $92.9 \%$ accuracy (precision $=0.949$, recall $=0.977$, F1 score $=0.963$ ). In these cases, we used the test set made by the first annotator as the ground truth. Finally, we compared the ground truth to the predicted bounding boxes. This performed almost as well as the annotators and reached a surprising 94.4\% accuracy (precision = 0.966 , recall $=0.977$, F1 score $=0.971)($ Fig. S1d $)$.

For the final classification result, we evaluated four different comparisons, following the same scheme as for the detection part. The intraexpert accuracies reached 97\% accuracy and $98.1 \%$ accuracy. The interexpert accuracy resulted in $90.5 \%$ accuracy. And the final comparison achieved $90.1 \%$ accuracy.

\section{In vitro experiments}

\section{Preparation of primary cultures}

The isolation of primary neurons was carried out according to Pacifici and Peruzzi [30] with some modifications. Primary cortical neurons were obtained from hHSPB1 transgenic mice and wild-type littermates on the 17th embryonic day. Briefly, embryos were washed in cold dissection medium, then the whole brain was extracted and was placed into an ice-cold Hibernate E medium (BrainBits, IL, USA). Cerebral cortices were isolated, while meninges were removed from the surface, and cortices were washed 3 times with Hibernate E, then were enzymatically digested with TrypLE (Gibco, Life Technologies, CA, USA) at $37^{\circ} \mathrm{C}$ for $10 \mathrm{~min}$. Next, the digested cortices were washed 3 times with Hibernate $\mathrm{E}$ and were homogenized in Neurobasal/B27 complete medium (Gibco, Life Technologies, CA, USA) containing $200 \mathrm{mM}$ Glutamax (Gibco, Life Technologies, CA, USA) with 18-G and 20-G needles (Braun, Germany). Finally, the suspension was diluted with Neurobasal/B27 complete medium, and cells were counted using Countess Automated Cell Counter (Thermo Fisher Scientific, MA, USA). Cells were plated on poly-D-lysine-coated 96-well plates at the density of $2 \times 10^{4}$ cells/well. Primary neurons were cultured in an incubator with $5 \% \mathrm{CO}_{2}$ and $37^{\circ} \mathrm{C}$ for 5 days.

Primary mouse glial cells were isolated and cultured as described in Lenárt et al. [31]. Briefly, forebrains without the cerebellum and the bulbus olfactorius were obtained from 4-day-old wild-type and hHSPB1 transgenic mice and placed into ice-cold PBS. Meninges were removed from the surface of the brains by using a fine forceps and sterile filter paper. Little pieces of cortices were pipetted to 50-ml tubes and then the tissue was mechanically dissociated using a long and thin needle (21G $43 / 4$, Braun, Germany). Isolated cells were plated onto uncoated T25 flasks (Corning Costar Co., MA, USA) and cultured in low-glucose Dulbecco's modified Eagle's medium (Gibco, Life Technologies, CA, USA) containing $10 \%$ fetal bovine serum (Sera Plus, Pan Biotech, Aidenbach, Germany) and gentamycin (Sigma-Aldrich Ltd, Budapest, Hungary) at the concentration of $50 \mu \mathrm{g} / \mathrm{ml}$ and were cultured until confluency before used for experiments. Medium was changed every 2 days.

Microglial cells were isolated from the surface of primary astroglia cultures [32]. Here astroglia were not passaged after reaching confluency but kept in the flask for longer periods. After 10 days, microglia cells migrate to the top of the confluent astroglia layer and start to round up on the surface. After gently tapping the flasks, the detaching microglia cells were collected and plated in astroglia culture medium onto poly-L-lysine-coated 96-well plates with a cell density of $2 \times 10^{4}$ cells/well. With this method, additional microglia cells were collected every second day for up to a week from the surface of the glial layer. Half of the medium was changed every day. Microglia cells received $50 \%$ glia cell-conditioned medium from day 3 and were cultured for 7 days before treatments.

\section{Assessment of the purity of the isolated primary cell cultures by immunostaining}

In order to determine the purity of primary neuron, astroglia, and microglia cultures, cells were passaged either to thin-bottom 96-well plates (Corning Costar Co., MA, USA) or to coverslips (VWR, PA, USA) and 
stained for specific markers. Glial cells and microglia were cultured on poly-L-lysine-coated glass coverslips and in 96-well plates along with neurons. Following the regular permeabilization and blocking steps (for this, see Methods' section "Fluorescent immunostaining"), cells were stained for specific markers. To evaluate the purity of the neuronal cultures, cells were double-labeled for neuronal marker microtubule-associated protein-2 (chicken anti-MAP2, Abcam, Cambridge, UK) and astrocyte marker GFAP (mouse anti-GFAP). Primary isolated astroglia cultures can be contaminated by microglia cells and vice versa; therefore, to assess the level of astroglia and microglia purity, we stained for GFAP and for the microglial marker IBA1 (goat anti-IBA1) (Table S1). The following secondary antibodies were used: Alexa Fluor-594conjugated donkey anti-chicken (Jackson ImmunoResearch Europe Ltd., Cambridgeshire, UK); Alexa Fluor-488-conjugated donkey anti-mouse (Life Technologies, Invitrogen, USA), Alexa Fluor-488-conjugated donkey anti-goat (Life Technologies, Invitrogen, USA), Dylight 549-conjugated goat anti-mouse (Jackson ImmunoResearch Europe Ltd., Cambridgeshire, UK) (Table S1). After mounting the samples (Fluoromount-G; Southern Biotech, AL, USA), pictures were taken at random positions (at least 3 images/sample), using fluorescent confocal laser scanning microscopes: Olympus Fluoview FV1000 (Olympus Life Science Europa GmbH, Germany) and Leica SP8 Confocal Microscope (Leica Microsystems, Germany). Cell nuclei were counted for both stainings and an image quantitation was performed where percentages of the marker positive cells were given normalized to the total cell number $(n=3-11)$.

\section{Treatments and collection of supernates}

Primary neurons were treated 5 days after the isolation with human TNF $\alpha$ and IL-1 $\beta$ (10 $\mathrm{ng} / \mathrm{ml}$ each) and 200 $\mathrm{mM} \mathrm{EtOH}$. The treatments on primary neurons were performed in 96-well plates in $50 \mu \mathrm{l} /$ well Neurobasal/ B27 complete medium (Gibco, Life Technologies, CA, USA). After $24 \mathrm{~h}$ of treatment, cell culture supernates were collected, an 3-3 wells were pooled, centrifuged at $510 \mathrm{~g}$ to remove any contaminating cells or debris, and then stored at $-80^{\circ} \mathrm{C}$. Meanwhile, cells were fixed with $4 \%$ PFA for $20 \mathrm{~min}$ and then stored in $1 \times \mathrm{PBS}$ at $4{ }^{\circ} \mathrm{C}$ for immunofluorescent staining.

All treatments and supernate collection from primary astroglia and microglia cells were performed similarly except that a lower concentration of EtOH $(50 \mathrm{mM})$ was used in glial cell culture medium. After the treatment, cell culture supernates were collected and stored at $80^{\circ} \mathrm{C}$. Cells were fixed with $3 \%$ PFA for $15 \mathrm{~min}$ at room temperature and then stored in PBS containing 0.1\% sodium azide at $4{ }^{\circ} \mathrm{C}$ for immunofluorescent staining.

\section{Fluorescent immunostaining}

To analyze the expression pattern of hHSPB1, PFA-fixed primary neuronal cells were permeabilized with $0.1 \%$ Triton X-100 for $10 \mathrm{~min}$. Cells were then blocked with 3\% BSA in PBS for $1 \mathrm{~h}$ and were incubated overnight with primary antibodies: chicken anti-MAP2 and rabbit antihHSPB1. Subsequently, the cells were incubated with secondary antibodies: Alexa Fluor-594-conjugated donkey anti-chicken and Alexa Fluor-488-conjugated goat antirabbit (Thermo Fisher Scientific, MA, USA) for $45 \mathrm{~min}$ (Table S1). Cell nuclei were labeled with DAPI (Sigma-Aldrich Ltd, Budapest, Hungary) at $0.5 \mu \mathrm{g} / \mathrm{ml}$ for $5 \mathrm{~min}$. The stainings were examined with a Leica SP8 Confocal Microscope (Leica Microsystems, Germany).

To further assess the hHSPB1 expression, microglia and astroglia cells were co-stained for their corresponding markers and for hHSPB1. After PFA fixation, permeabilization was carried out with $0.2 \%$ Triton X-100 in PBS for $10 \mathrm{~min}$, followed by blocking with $2 \%$ normal horse serum or $5 \%$ normal goat serum. Primary antibodies were incubated overnight: goat anti-IBA1, mouse anti-GFAP, rabbit anti-hHSPB1. Incubation with secondary antibodies Alexa Fluor-488-labeled donkey anti-goat, Cy3-labeled sheep antirabbit (Sigma-Aldrich Ltd, Budapest, Hungary), Dylight A488-conjugated goat anti-rabbit (Jackson ImmunoResearch Europe Ltd., Cambridgeshire, UK), and Dylight 549-conjugated goat anti-mouse lasted for $1 \mathrm{~h}$. Hoechst dye 33342 was used for nucleus staining (Table S1). After mounting the samples (Fluoromount-G; Southern Biotech, AL, USA), staining was examined by Olympus Fluoview FV1000 confocal laser scanning microscope (Olympus Life Science Europa GmbH, Hamburg, Germany).

The images were analyzed using the Matlab software (MathWorks, Natick, MA, USA). An average threshold was calculated for each staining using the images from the transgenic and wild-type animals. The binary images were determined by using the corresponding average threshold. Objects with a size of less than 4 pixels were eliminated to reduce any false structures. The colocalization was received by taking overlapping structures of the corresponding binary images. The overall intensities were calculated using the final binary images as masks on the original grayscale images. The intensity values gained from astrocytes were normalized to the pixel numbers from where the intensity data was collected $(n=9-12$ for GFAP in TG astrocytes; $n=14-15$ for GFAP in WT astrocytes; $n=10-11$ for hHSPB1 in TG astrocytes). Due to the presence of astrocytes in neuronal culture, which also highly express the transgene, HSPB1 intensity values were obtained from neurons and normalized to the pixel number of neuronal marker MAP2 $(n=12)$. 
Enzyme-linked immunosorbent assay analysis of cell culture supernates

Concentrations of released hHSPB1 and TNFa in the supernate of all three primary isolated cell cultures were quantified using human HSPB1 ELISA kit (Enzo Life Sciences, NY, USA) and mouse TNF alpha ELISA kit (Life Technologies, Invitrogen, USA). Briefly, cell culture supernates were collected and additional sample diluents were added. For all cell types, samples from multiple experiments were tested to create biological and technical parallels $(n=2-3)$. We used $47 \mu \mathrm{l}$ sample/well for the mouse TNF $\alpha$ assay, and $95 \mu \mathrm{l}$ sample/well for the hHSPB1 assay. After the addition of supernates to the wells, the assay was carried out according to the manufacturer's instructions.

\section{Statistical analysis}

All data obtained in this experiment are expressed as mean \pm SEM. Statistical analysis was performed by twoway analysis of variance (2-way ANOVA) followed by Tukey post hoc test using the OriginPro8 software (OriginLab, MA, USA) and unpaired two-tailed $t$ test using GraphPad Prism software (GraphPad Software Inc., San Diego, CA, USA). Data were considered statistically significant at $p<0.05$.

\section{Results}

\section{Expression pattern of transgenic human HSPB1 in the mouse brain}

The expression of the transgenic hHSPB1 was studied in the brain of 7-day-old mice using various methods. Whole-brain homogenates were analyzed by Western blotting (Fig. 1a, b) and RT-PCR (Fig. 1c). High expression of transgenic hHSPB1 was detected in transgenic brains both at the protein and mRNA levels. hHSPB1 expression increased $24 \mathrm{~h}$ after ethanol treatment, especially in the hippocampal region and in the retrosplenial cortex, as shown by our immunohistochemical analysis (Fig. 1d). The cell-specific expression pattern of the transgene was also monitored using NEUN-hHSPB1, GFAP-hHSPB1, and IBA1-hHSPB1 fluorescent double immunostainings (Fig. 1e). The NEUN-hHSPB1 double immunostaining revealed the presence of the transgenic hHSPB1 protein in neuronal cells. The GFAP immunolabeling colocalized with hHSPB1, indicating that astrocytes also expressed the transgene. IBA1 immunoreactive microglia, however, showed no remarkable colocalization with hHSPB1.

\section{Differences in the gene expression of pro- and anti- inflammatory cytokines in hHSPB1 transgenic and wild- type mouse brains}

The expression level of cytokines as molecular markers of inflammation was studied using semiquantitative RT-
PCR at different time points after ethanol treatment. In nontreated transgenic mice, hHSPB1 overexpression alone did not affect significantly the mRNA level of the studied pro-inflammatory cytokines, whereas it contributed to a marked increase in gene expression after ethanol treatment. In response to ethanol, the level of Tnf mRNA was elevated as early as $7 \mathrm{~h}$, showing a threefold (297\%) increase in wild-type and a fourfold (413\%) increase in hHSPB1-overexpressing animals compared to saline-treated controls (Fig. S2a). One day later, Tnf expression started to decrease in the wild-type animals but still maintained a twofold (229\%) increase compared to the nontreated controls (Fig. 2a). However, in transgenic animals, we detected a further elevation in the level of Tnf mRNA, reaching a tenfold (1031\%) increase in expression compared to untreated wild-types (Fig. 2a). Similar changes were found in the expression level of Il1b: it also increased in response to the ethanol treatment; however, this had happened at a later time point. One day after the treatment, it showed a twofold increase in the wild-type animals, while in the transgenic ones, we detected a much higher, a more than sevenfold increase (706\%, Fig. 2b). Another inflammation-inducing cytokine, Il6, also showed a similar, but nonsignificant trend towards increased expression in ethanol-treated transgenic mice (Fig. 2c). Contrarily, in the case of antiinflammatory cytokines, we observed changes only in the mRNA level of $T g f b$, whereas the expression levels of $I l 10$ and $I l 4$ remained unchanged in response to hHSPB1 overexpression as well as after ethanol treatment (Fig. $2 \mathrm{~d}-\mathrm{f}$ ). In the nontreated group, the expression level of the $T g f b$ was twice as high in hHSPB1-overexpressing animals as in wild-type ones $24 \mathrm{~h}$ after saline injection (Fig. 2d). One day after ethanol administration, the expression level of $T g f b$ showed a significant increase in wild-type animals (233\%), whereas in transgenic mice, no further increase was detected. After 1 week, the gene expression levels of the investigated cytokines decreased to their original levels both in the wild-type and in the transgenic brains (Fig. S2a-c).

\section{Altered microglial activation in hHSPB1 transgenic brains}

The expression level of the microglia-specific allograft inflammatory factor 1 (Aif1, gene encoding protein IBA1) was analyzed using RT-PCR. In the saline-treated group, hHSPB1 transgenic animals had more than two times higher Aif1 mRNA level (261\%) compared to wildtypes after $24 \mathrm{~h}$ (Fig. 3a). Ethanol administration increased the Aif1 mRNA levels in wild-type animals (205\%) 1 day after treatment, while no further increase was observed in the ethanol-treated transgenic pups (Fig. 3a). Morphological changes of microglial cells were studied using IBA1 immunostaining which was evaluated by deep learning methods. The highest proportions 

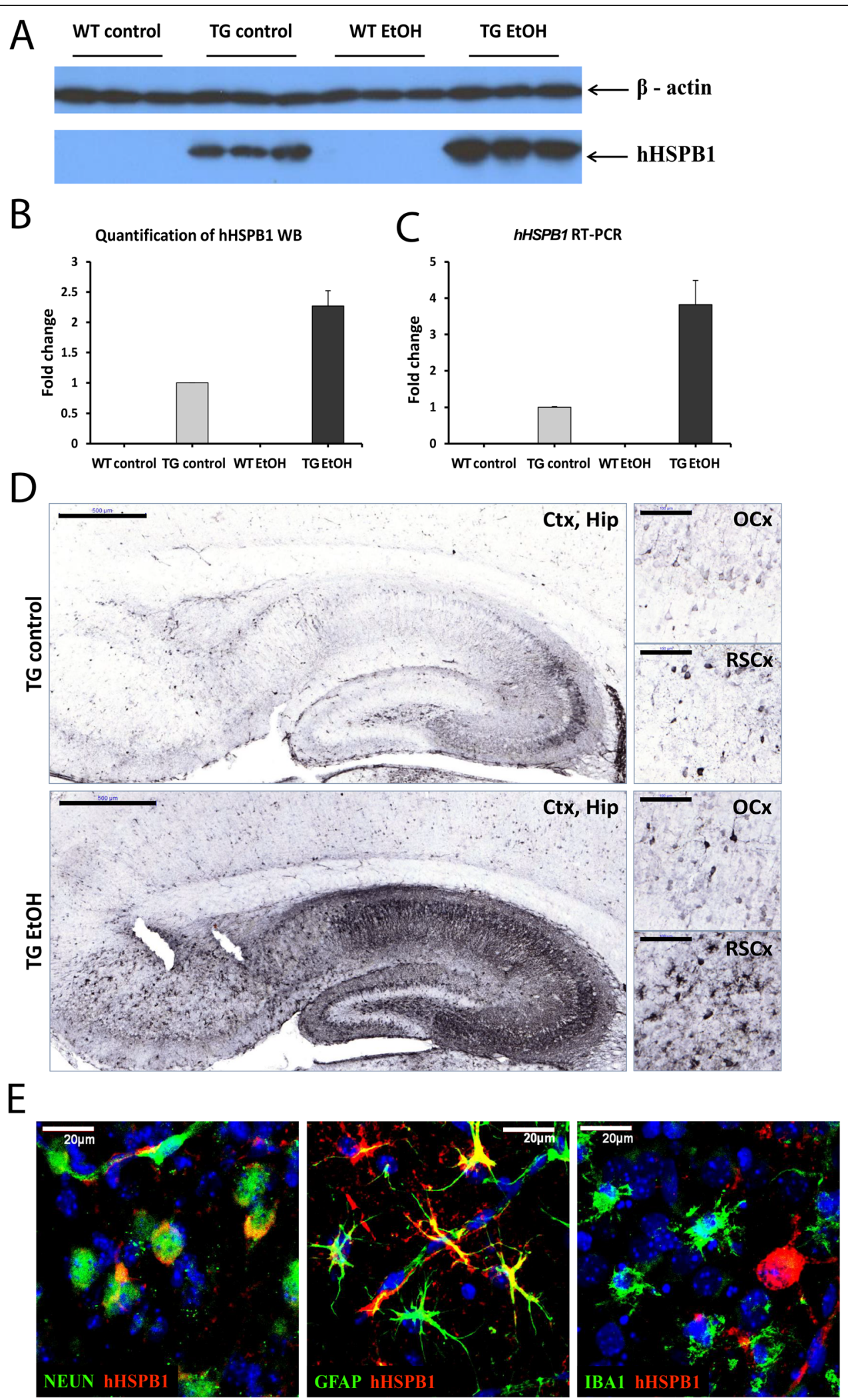

Fig. 1 (See legend on next page.) 
(See figure on previous page.)

Fig. 1 Transgene expression analysis in the brain. a hHSPB1 protein expression level was measured in whole-brain homogenates $24 \mathrm{~h}$ after ethanol treatment using Western blot. Mouse $\beta$-actin was used as an internal control. $\mathbf{b}$ Quantification of the results was performed using the open-source ImageJ software. Fold changes were correlated with the transgenic control group. Data are represented as mean \pm SEM; $n=3$ mice per group. c Relative expression of the transgenic hHSPB1 $24 \mathrm{~h}$ after ethanol treatment was studied in the brain using RT-PCR. Fold changes were correlated with the transgenic control (nontreated) group. Values are presented as mean \pm SEM; $n=8$ mice per group. $\mathbf{d}$ hHSPB1 immunolabeling on sagittal brain sections of saline- and ethanol-treated transgenic mice $24 \mathrm{~h}$ after treatment. Scale bar: $500 \mu \mathrm{m}$ (Ctx, Hip) and $100 \mu \mathrm{m}$ (OCx and RSCx). Ctx cortex, Hip hippocampus, RSCx retrosplenial cortex, OCx occipital cortex. e Fluorescent double immunohistochemistry on sagittal brain sections showing cell type-specific expression of the transgene in the cortical region. Scale bar: $20 \mu \mathrm{m}$. Red: hHSPB1; green: IBA1/NEUN/GFAP; blue: DAPI

of resting microglial cells with highly ramified, fine processes were detected in the brain of the untreated animals; however, there were some differences observed between the wild-type and transgenic samples (Fig. 3b, c). Control transgenic animals had significantly smaller amounts of activated (intermediate and amoeboid) and higher amounts of resting microglia in the thalamus, and similar, albeit nonsignificant, changes were observed in the other brain regions as well (Fig. 3c; see Table S3 for the statistical analysis of the proportion of activated microglia). In contrast to saline treatment, $24 \mathrm{~h}$ after ethanol administration, we detected a high proportion of activated, hypertrophied microglia with increased cell bodies and shorter, thicker processes in the striatum as well as in the parietal and frontal parts of the cortex, whereas amoeboid microglia were only present in a smaller percentage in these areas. Contrarily, in the retrosplenial and occipital cortical regions or in the thalamus and in the hippocampus, we found a particularly high proportion of amoeboid microglia cells along with

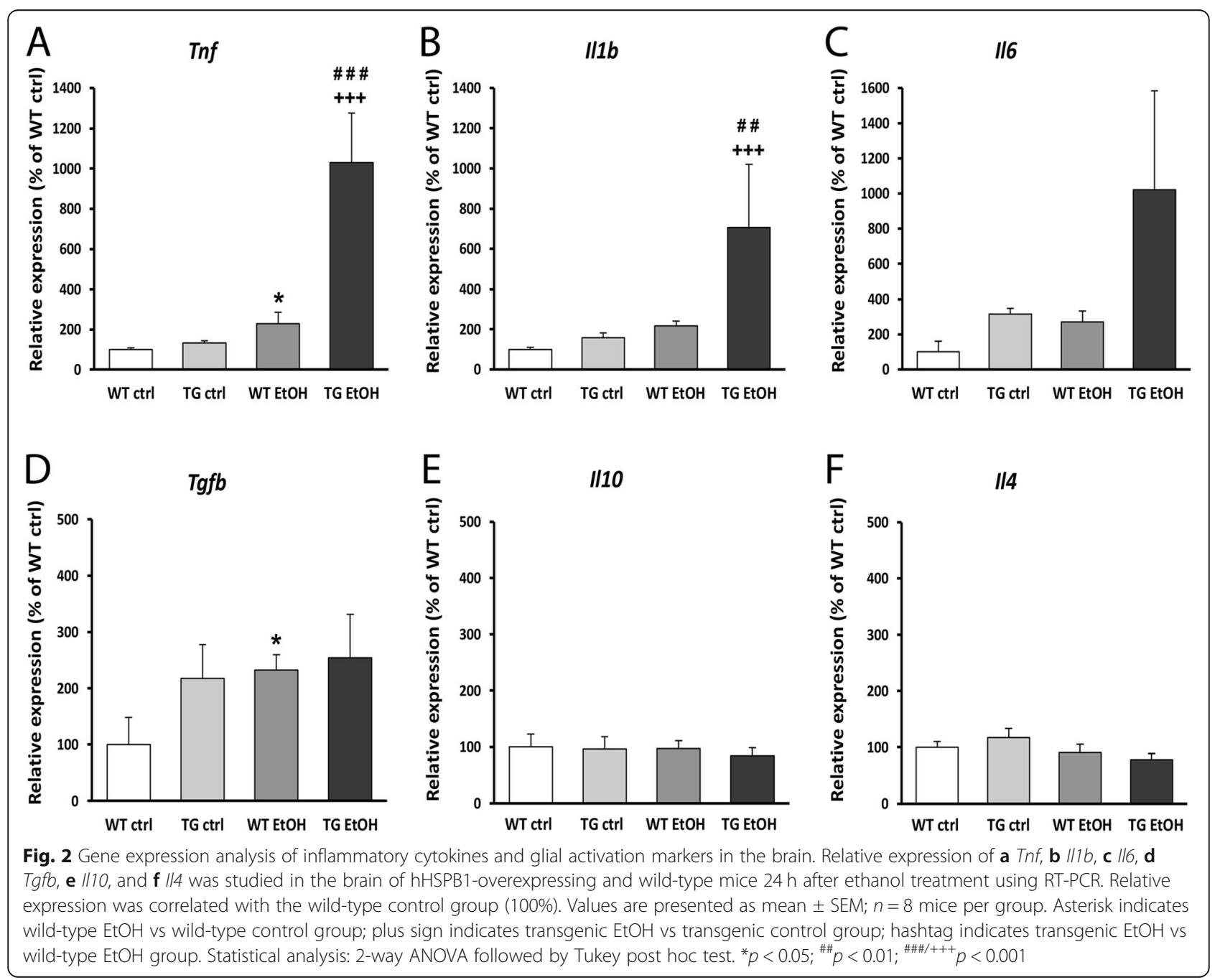




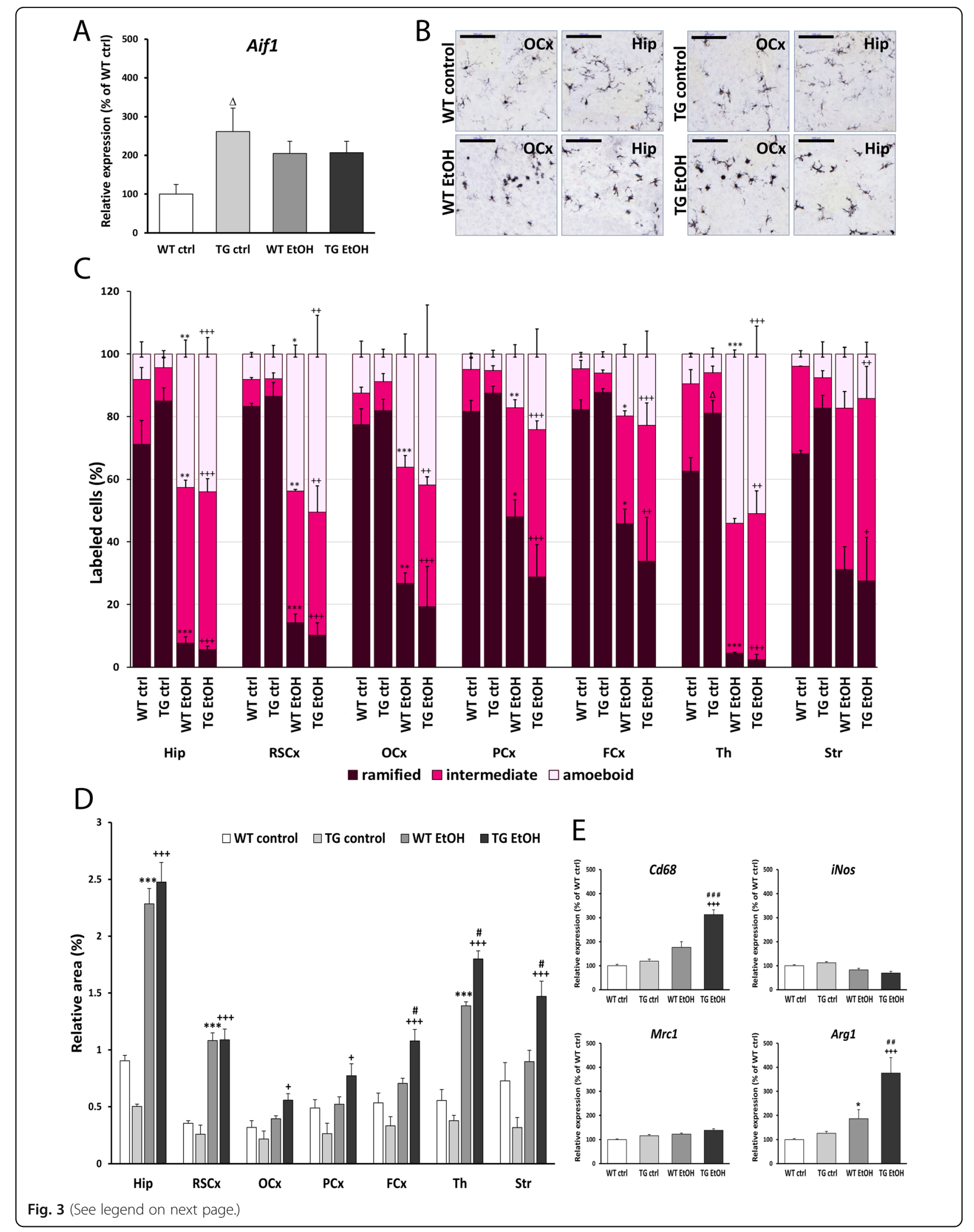


(See figure on previous page.)

Fig. 3 Activation of microglia $24 \mathrm{~h}$ after ethanol treatment in the brain of transgenic and wild-type mice. a Relative expression of Aif in the brain of hHSPB1-overexpressing and wild-type mice $24 \mathrm{~h}$ after ethanol treatment. Relative expression was correlated with the wild-type control group (100\%). $n=8$ mice per group. b Morphological changes of microglia $24 \mathrm{~h}$ after EtOH treatment on sagittal brain sections of hHSPB1overexpressing and wild-type mice. Microglia with different morphologies were visualized with IBA1 immunostaining using the peroxidase method. Scale bar: $100 \mu \mathrm{m}$ (OCX and Hip). c Proportions of IBA1+ microglia in different brain regions according to their morphology. $\mathbf{d}$ Quantification of IBA1 immunoreactive areas. Results are given in percentage of the immunopositive areas compared to the outlined brain areas (relative area). $n=3$ mice per group, 3 sections per animal. e Relative expression of M1 microglia markers (Cd68, iNos) and M2 microglia markers (MrCl, Arg 1) in the brain of hHSPB1-overexpressing and wild-type mice $24 \mathrm{~h}$ after ethanol treatment. Relative expression was correlated with the wild-type control group (100\%). $n=8$ mice per group. Values are presented as mean \pm SEM. Asterisk indicates wild-type EtOH group vs wild-type control group; plus sign indicates transgenic EtOH group vs transgenic control group; hashtag indicates transgenic EtOH group vs wild-type EtOH group; white triangle indicates transgenic control vs wild-type control group. Statistical analysis: 2-way ANOVA followed by Tukey post hoc test. ${ }^{* / \# /+/ \Delta} p<0.05 ;{ }^{* * / \# /+++} p<0.01 ; * * * / \# \# /++++p<0.001$. Ctx cortex, Hip hippocampus, RSCx retrosplenial cortex, OCx occipital cortex, PCx parietal cortex, FCx frontal cortex, Th thalamus, Str striatum

the intermediate ones (Fig. 3b, c). The proportion of intermediate and amoeboid microglia was significantly higher in all brain areas of the ethanol-treated mice compared to those of the control animals; however, the transgenic group showed a nonsignificant trend of increased proportion of activated microglia compared to ethanoltreated wild-type one (Fig. 3c). In parallel, the IBA1 coverage was more extensive in both ethanol-treated transgenic and wild-type brains compared to control ones. This increase in extent after ethanol treatment was more pronounced in the transgenic animals with significant differences in the frontal cortical, thalamic, and striatal regions (Fig. 3d). To determine the characteristic features of the activated microglia cells, we analyzed the expression of M1 (pro-inflammatory) and M2 (anti-inflammatory) phenotypic markers. In the case of M1 markers, only Cd68 showed notable changes; as in response to ethanol treatment, a significant threefold increase (312\%) was detected in hHSPB1 transgenic animals, whereas only a slight increase was observed in wild-type animals. Interestingly, the level of the other M1 marker, the inducible nitric oxide synthase (iNos), remained unchanged after treatment (Fig. 3e). Similar changes were observed for M2 markers. One of the markers, the mannose receptor C-type 1 (Mrc1), also remained unchanged, while the other one, arginase 1 (Arg1), was nearly doubled in wild-type animals (186\%) and showed an even significantly higher, almost fourfold increase $(375 \%)$ in hHSPB1 transgenic animals after ethanol treatment (Fig. 3e). One week after the treatment, microglial cells returned to their resting state-like morphology (Fig. S3a). Although Aif1 mRNA levels were approximately the same in each group, 1 week after treatment, the IBA1covered area remained smaller in most brain areas of ethanol-treated wild-type animals compared to those of the control wild-type group (Fig. S2d and Fig. S3b).

\section{Ethanol-induced activation of astrocytes in the brain of hHSPB1-overexpressing mice}

In the next step, we studied the mRNA expression levels of Gfap and Vim, two intermediate filament proteins of astrocytes used as markers of reactive astrogliosis. The level of Gfap mRNA was increased $24 \mathrm{~h}$ after ethanol treatment both in the wild-type and transgenic groups, showing a fourfold increase in the transgenic group (440\%) and a twofold increase in the wild-type one (196\%, Fig. 4a). In contrast, the expression level of Vim mRNA did not change in the wild-type group and only slightly increased in the transgenic group in response to ethanol treatment (Fig. 4a). The level of both markers returned to the level of the control group 1 week after the treatment (Fig. S2e-f). The expression pattern of GFAP at the protein level and morphological changes of astrocytes was studied using immunohistochemistry. No significant change was detected in the morphology of astroglia $24 \mathrm{~h}$ after ethanol treatment (Fig. S4a-b). One week later, however, an increased GFAP immunoreactivity and hypertrophic astrocytes were detected in different brain regions both in wild-type and transgenic mice in response to ethanol treatment (Fig. 4b, c). Quantification of the immunostained area revealed a strong enhancement in GFAP coverage throughout the brain 1 week after ethanol treatment. A significant difference between ethanol-treated wild-type and transgenic mice was found in the striatum and in the parietal cortex (Fig. 4b).

\section{Changes in the level of ethanol-induced apoptosis in HSPB1-overexpressing transgenic mice}

In order to detect programmed cell death in the brain sections of mice, we analyzed the amount of fragmented DNA using TUNEL assay. In the brain of the control, saline-treated animals, a very low level of apoptosis was observed, while the number of TUNEL-positive cells was dramatically increased $24 \mathrm{~h}$ after ethanol treatment (Fig. 5a). For quantitative comparison, the TUNEL-positive cells were counted in fifteen areas of each brain section of each group. The statistical analysis revealed a significant increase in the number of apoptotic cells in most of the studied brain regions following ethanol treatment. The highest number of apoptotic cells was found in the 


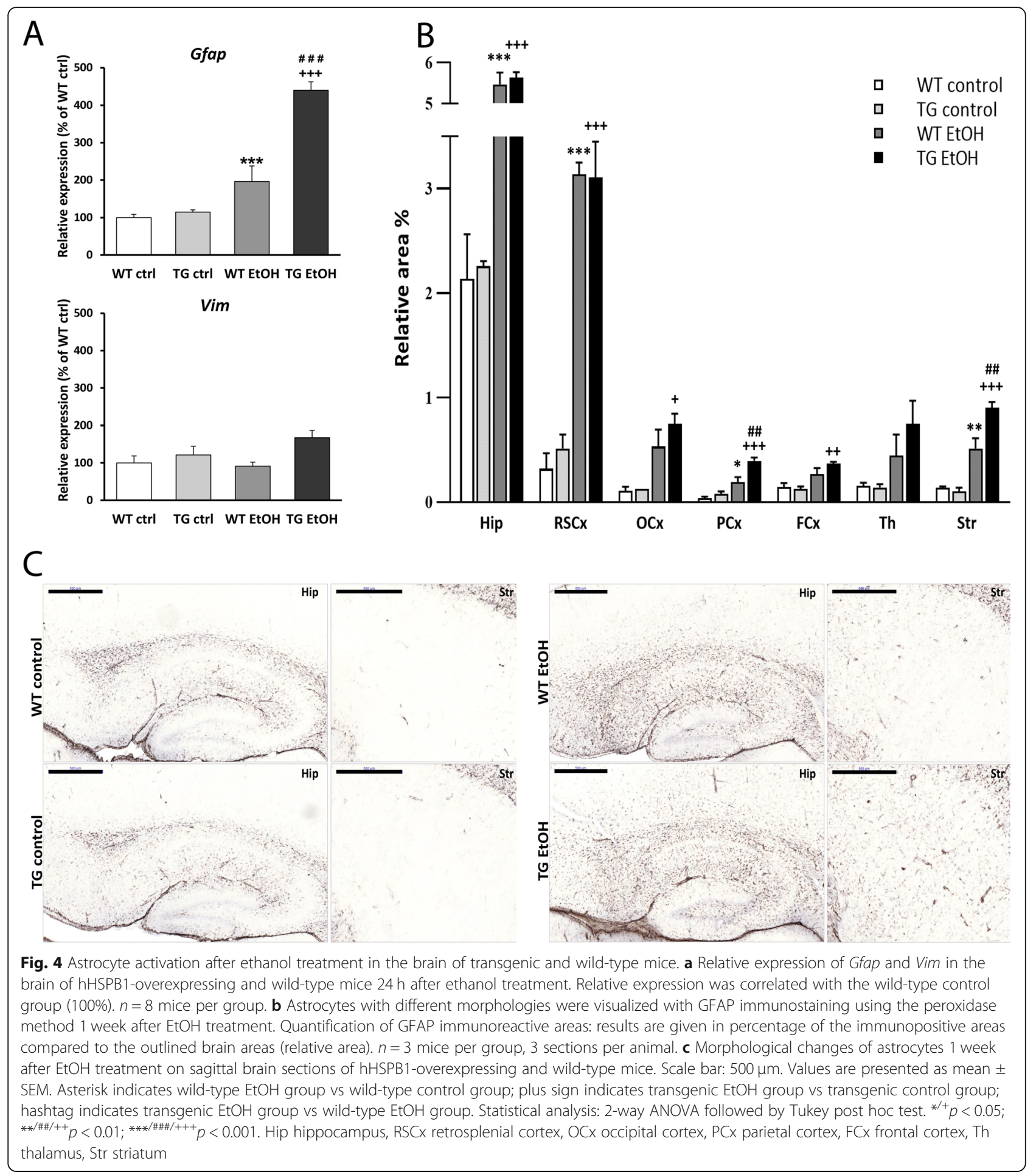

cortex, thalamus, striatum, and midbrain, whereas a lower level of apoptosis was detected in the hippocampus. In the cerebellum, a high level of physiological cell death was visible, but the number of apoptotic cells remained similar after ethanol treatment (Fig. 5b). Comparing wild-type and hHSPB1-overexpressing animals, a slightly lower number of apoptotic cells was found in the brain of the transgenic animals; however, the difference was not statistically significant (Fig. 5b).

These in vivo results (summarized in Fig. S9) served as a basis for our subsequent in vitro experiments where we aimed to characterize the specific cell type that could be responsible for the aforementioned alteration of immune regulation triggered by the overexpression of hHSPB1. 


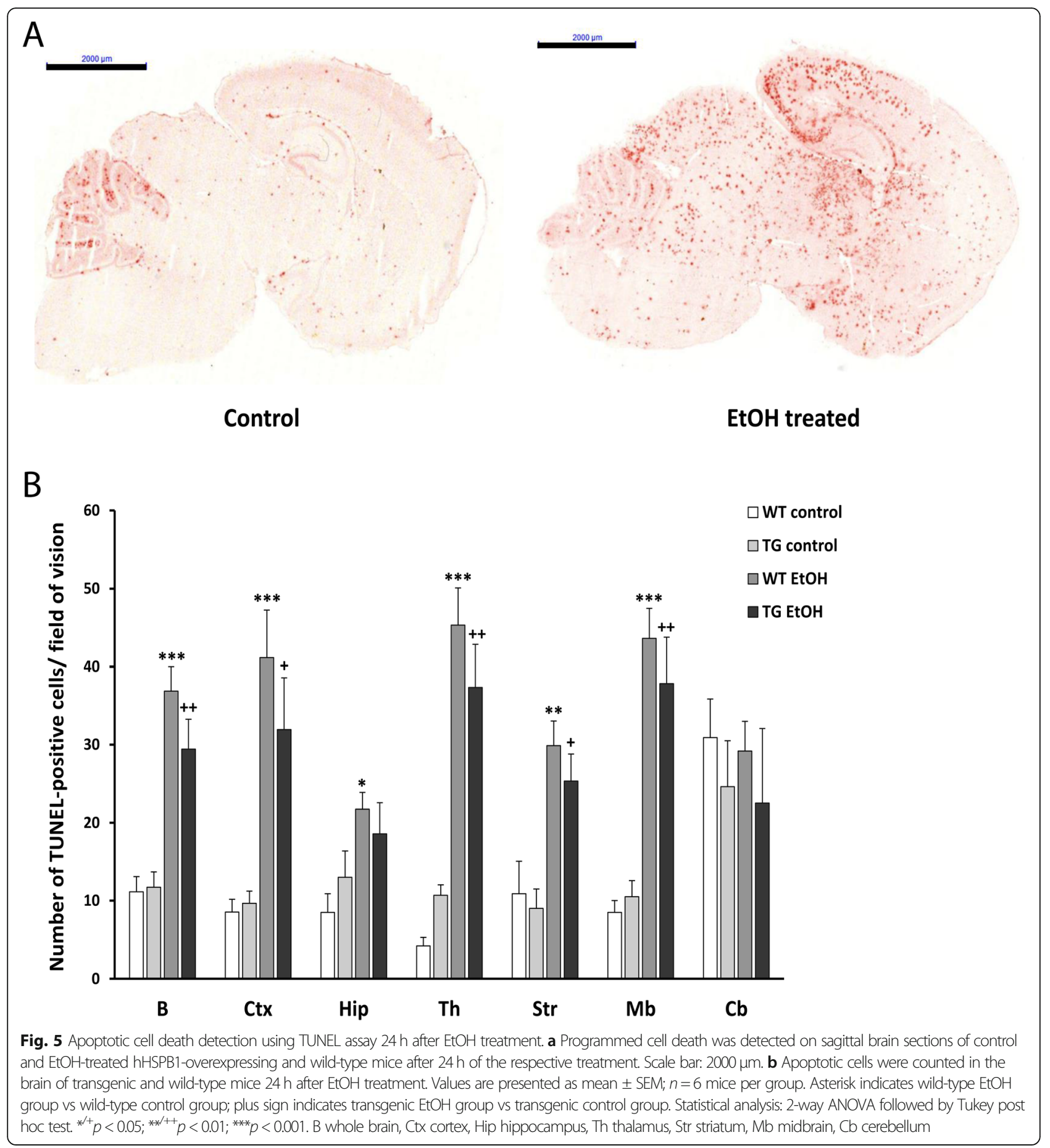

Characterization of primary neuronal, astrocyte, and microglial cell cultures derived from hHSPB1 and wildtype mice

Primary neuron, astrocyte, and microglia cultures were prepared from the brain of hHSPB1 transgenic animals and from their wild-type littermates. First, the purity of the primary cultures was assessed by fluorescent immunohistochemistry, examining the presence of microglial
(IBA1), astrocyte (GFAP), and neuronal (MAP2) markers. IBA1-GFAP double staining of the primary microglia culture confirmed that its purity exceeded $93 \%$ (Fig. 6a). We used the same double staining to determine the purity of the primary astrocyte culture, which indicated that the microglia cells were present at the cell culture at a proportion of $22 \%$ (Fig. 6b). The primary neuronal culture was labeled with MAP2 and GFAP 


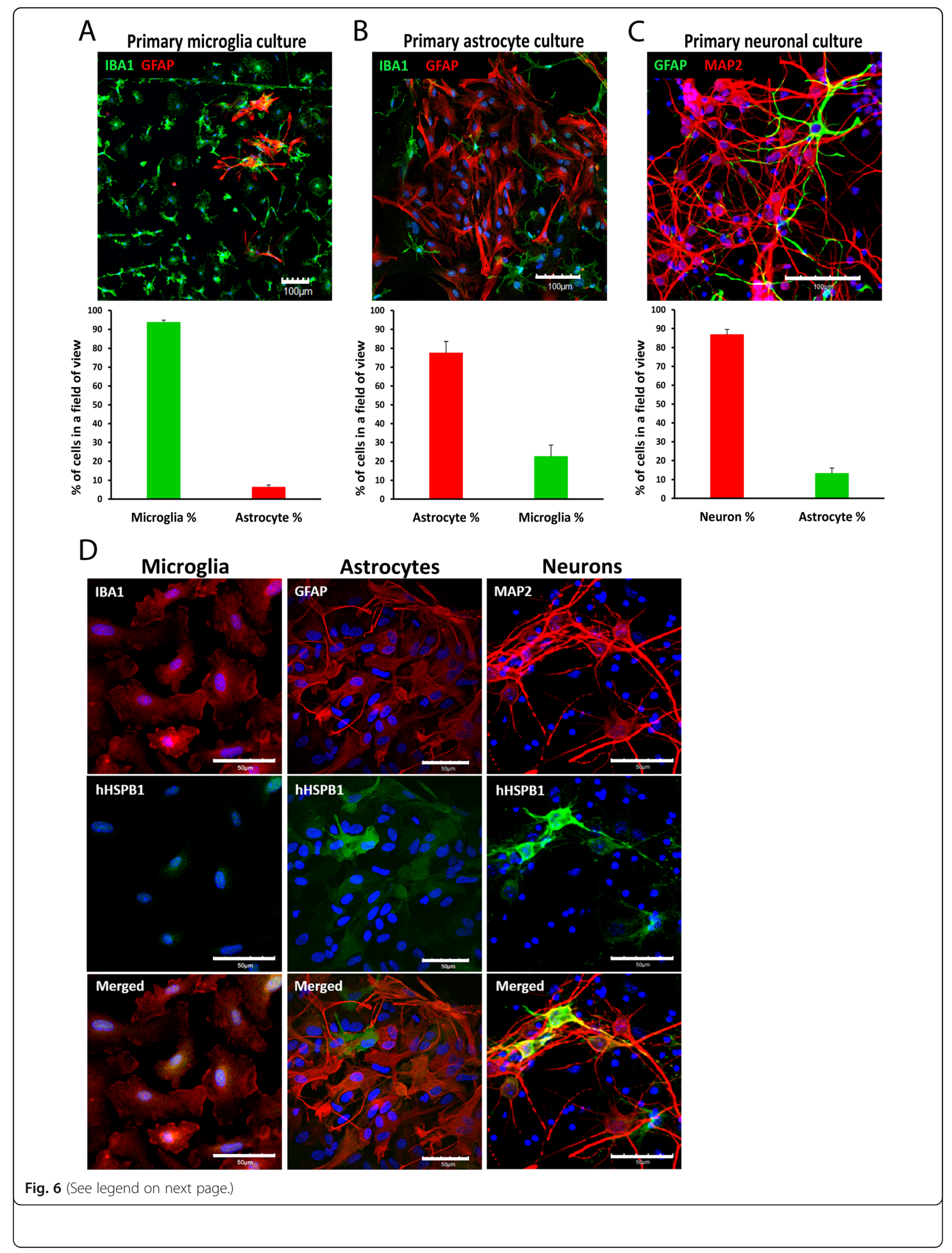


(See figure on previous page.)

Fig. $\mathbf{6}$ Characterization of the established primary cell cultures. Evaluation of the purity of primary a microglia, $\mathbf{b}$ astrocyte, and $\mathbf{c}$ neuronal cultures using fluorescent immunohistochemistry and detection of the presence of microglial (IBA1—green), astrocytic (GFAP_green/red), and neuronal (MAP2 - red) markers. Cell nuclei were counted for cell-specific markers and percentages compared to the total cell number are given. Scale bar: $100 \mu \mathrm{m}$. Values are presented as mean \pm SEM; $n=3-11$. d Analysis of the expression pattern of the transgenic hHSPB1 protein in primary cell cultures using double immunofluorescent staining. Scale bar: $50 \mu \mathrm{m}$. Green: hHSPB1; red: IBA1/MAP2/GFAP; blue: DAPI

antibodies, which showed that more than $85 \%$ of the cells were neurons in the culture (Fig. 6c). We also analyzed the expression pattern of the transgene in the primary cell cultures using hHSPB1-MAP2, hHSPB1GFAP, and hHSPB1-IBA1 fluorescent immunostainings. The colocalization of the cell-specific markers with hHSPB1 was similar to what was observed in the brain sections earlier. We found that hHSPB1 colocalized with MAP2 and GFAP, but was rarely detectable together with IBA1, indicating that the transgenic hHSPB1 protein was mainly expressed by primary neurons and astrocytes (Fig. 6d).

\section{Effect of ethanol and cytokine treatment on the TNFa production of primary neuron, astrocyte, and microglia cultures}

Primary cell cultures were treated with ethanol in order to investigate the effect of alcohol on different cell types of the brain. Ethanol can induce inflammatory processes in the brain directly by itself and also by its metabolites $[33,34]$, and it can also contribute to cerebral inflammation indirectly by disrupting the integrity of the blood-brain barrier, subsequently promoting the infiltration of peripheral immune cells to the brain [35]. Considering this affiliation of ethanol treatment with inflammation, we also treated our model with proinflammatory cytokines in order to model the inflammatory milieu connected with the presence of ethanol. The effective treatment concentrations of the cytokine and ethanol were selected based on the data available in the literature [21,36] and on corresponding cell viability measurements. We used a concentration of $50 \mathrm{mM}$ ethanol for astrocytes and microglia cells and $200 \mathrm{mM}$ for neurons. During the cytokine treatment, the combination of $10 \mathrm{ng} / \mathrm{ml}$ human TNF $\alpha$ and $10 \mathrm{ng} / \mathrm{ml}$ human IL$1 \beta$ was applied to all cell types (see Supplementary material and Fig. S5 for further details).

In order to analyze which cell type is the main source of TNF $\alpha$ and to confirm whether hHSPB1 regulates cytokine production in different brain-derived cells in our model, we performed an enzyme-linked immunosorbent assay (ELISA) assay specific against TNF $\alpha$ using the supernates

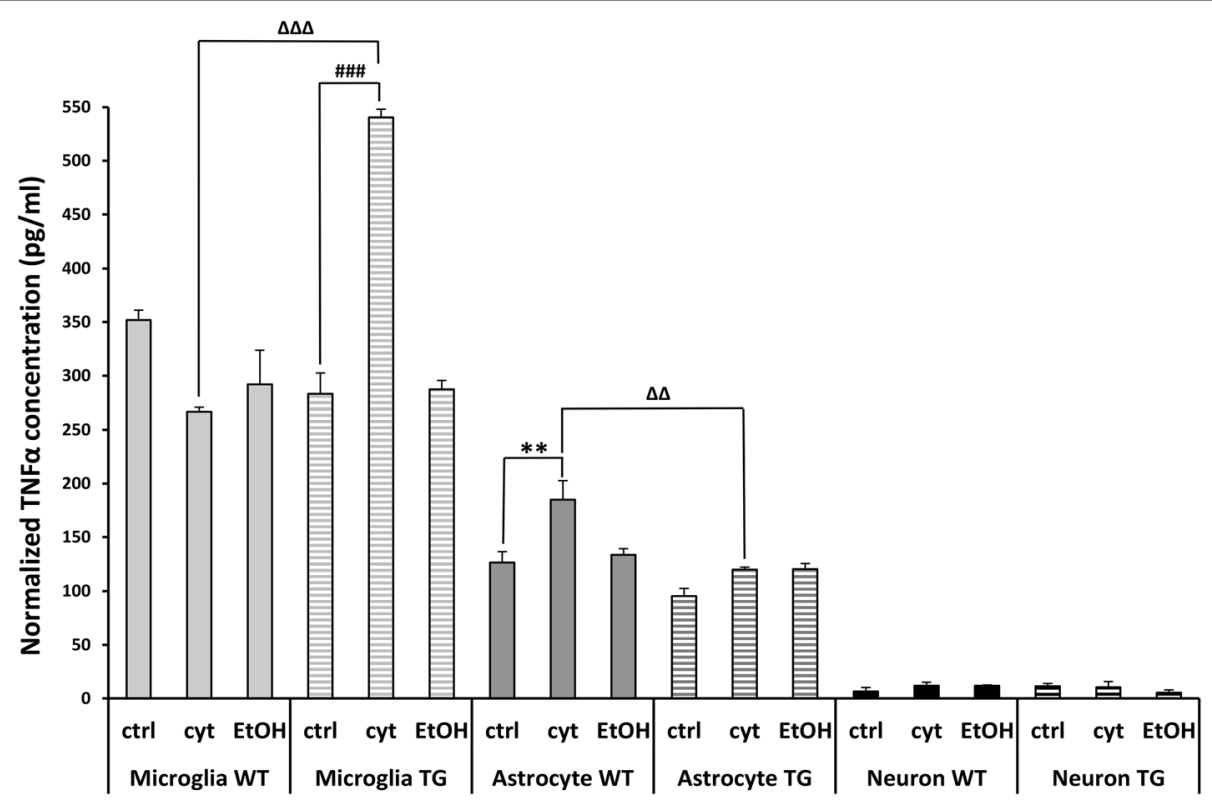

Fig. 7 Quantification of TNFa production of primary cell cultures. Twenty-four hours after EtOH and cytokine treatment, cell culture supernates were collected. Concentrations of released TNFa $(\mathrm{pg} / \mathrm{ml})$ in the supernate of all the three isolated primary cell cultures were quantified using ELIS A. TNFa concentrations were normalized to cell number of microglia and to RTCA values of astrocytes and neurons. crtl untreated control, EtOH ethanol treatment, cyt cytokine treatment. Values presented are means $\pm \mathrm{SEM} ; n=2-3$. Asterisk indicates wild-type cytokine vs wild-type control group; hashtag indicates transgenic cytokine vs transgenic control group; white triangle indicates transgenic cytokine vs wild-type cytokine group. Statistical analysis: 2-way ANOVA followed by Tukey post hoc test. ${ }^{* / \Delta \Delta_{p}}<0.01$; ${ }^{\# \# / \Delta \Delta \Delta_{p}<0.001}$ 
from primary cultures. The TNF $\alpha$ concentration values were normalized to the corresponding cell viability measurements: to cell number for microglia and to RTCA values for astrocytes and neurons. Under control conditions, TNF $\alpha$ was detectable in the highest proportion in the supernates of glial cells. Microglial cells had almost three times higher basal expression of TNF $\alpha$ (TG group: $283 \mathrm{pg} / \mathrm{ml}$; Wt group: $351 \mathrm{pg} / \mathrm{ml}$; columns $1-6$, Fig. 7) compared to astrocytes (TG group: $95 \mathrm{pg} / \mathrm{ml}$; Wt group: $126 \mathrm{pg} / \mathrm{ml}$; columns 7-12, Fig. 7), indicating that microglia are the primary source of TNF $\alpha$ in the brain. Cytokine treatment significantly increased the release of TNF $\alpha$ from hHSPB1-expressing transgenic microglial cells compared to nontreated transgenic cells (from 283 to $540 \mathrm{pg} / \mathrm{ml}$ ). However, no such effect was observed in wild-type microglia. This indicates that the overexpression of hHSPB1 might modulate the release of TNF $\alpha$ since microglia from hHSPB1 transgenic animals expressed a significantly higher level of TNF $\alpha$ after cytokine treatment than the cytokine-treated wild-type group $(540 \mathrm{pg} / \mathrm{ml}$ and $266 \mathrm{pg} /$ $\mathrm{ml}$, respectively; Fig. 7). Contrarily, after cytokine treatment, TNF $\alpha$ production was significantly higher in wildtype astrocytes compared to the control group; however, it remained unchanged in the hHSPB1 transgenic cells resulting in a significant difference between the two genotypes upon cytokine treatment (WT: $184 \mathrm{pg} / \mathrm{ml}$ and TG: $120 \mathrm{pg} / \mathrm{ml}$, respectively; Fig. 7). Surprisingly, ethanol treatment only did not elicit a response from either glial cell type (Fig. 7). In the supernates of primary neurons, a very low TNF $\alpha$ presence was observed under control conditions (Fig. 7), and no change was seen in response to the various treatments.

\section{Effect of cytokine and ethanol treatments on extracellular and intracellular hHSPB1 levels}

To determine whether extracellular or intracellular hHSPB1 elicits the proposed inflammation-regulating effects, we examined the level of hHSPB1 protein in the cell culture supernates by ELISA and in the cells by immunohistochemistry. The results of the human HSPB1 ELISA showed that no detectable amount of hHSPB1 was released by any of the analyzed cell types, upon treatment or under control conditions (Fig. S6). As microglial cells did not express an appreciable amount of intracellular hHSPB1 even after treatment (Fig. S7), we analyzed the changes in the intracellular level of the transgenic protein only in primary astrocytes and neurons. We found that the expression level of the transgene within primary neurons and astrocytes was influenced by the different treatments. In the case of primary neurons, the size of the MAP2-positive area was not altered by cytokine treatment, while it was significantly reduced by the ethanol treatment in both transgenic and wild-type neuronal cultures (to $41 \%$ and $46 \%$, respectively) (Fig. 8a, b, Fig. S8a-b). In HSPB1 transgenic neurons, the fluorescence intensity corresponding to hHSPB1 remained unchanged after ethanol treatment, whereas it showed a moderate, but not significant increase after the cytokine treatment (Fig. 8a, c). In astrocyte cells, treatment with cytokines or ethanol elevated the intracellular GFAP levels. The cytokine treatment resulted in a significant increase in GFAP intensity in both transgenic and wild-type cells (to $138 \%$ and to $131 \%$, respectively), whereas after the exposure to ethanol, a significant change was detected only in the transgenic astrocytes (136 \%), but not in wild-type ones (Fig. 8d, e, Fig. S8c-d). In transgenic astrocytes, the level of hHSPB1 expression elevated significantly to $475 \%$ well above the control's baseline expression after cytokine treatment and up to $205 \%$ after the ethanol treatment (Fig. $8 d$, f).

\section{Discussion}

The neuroprotective role of HSPB1 in both acute and chronic neurodegenerative disorders has been previously demonstrated by multiple research groups including ours [20,37, 38]. In parallel, a number of studies, using either mouse models of peripheral inflammation or cell cultures, revealed the regulatory function of HSPB1 in inflammation. In these studies, HSPB1 was affiliated with either pro- or anti-inflammatory functions in a contextdependent manner [2]. By expanding our knowledge on HSPB1 function as an immunoregulatory factor, it could become a potential therapeutic target for fine-tuning neuroinflammation, which is a common pathological characteristic of most neurological disorders. Therefore, in the present study, we investigated the immunoregulatory role of hHSPB1 in neuroinflammation using our previously established hHSPB1-overexpressing mouse model, as well as using primary cell cultures isolated from the brain of these transgenic animals.

The brain is extremely sensitive to the cytotoxic effect of ethanol during synaptogenesis, the period of brain development when it undergoes rapid growth. Neurons are vulnerable to ethanol-induced damage at this period to such an extent that even a single day of alcohol treatment can lead to neurodegeneration in the brain. Ikonomidou and colleagues [21] demonstrated that maintaining the blood ethanol concentration in 7-day-old rat pups above a toxic threshold $(200 \mathrm{mg} / \mathrm{dl})$ for at least $4 \mathrm{~h}$ triggers apoptotic cell death detectable $24 \mathrm{~h}$ after the treatment. Besides apoptosis, acute ethanol overdose has been demonstrated to trigger microglia and astrocyte activation in different animal models, so it is also suitable for investigating neuroinflammation [39]. Therefore, in this study, the same experimental arrangement was used to induce brain damage in wild-type and hHSPB1-overexpressing transgenic mice. In our transgenic animals, the human HSPB1 gene expression is driven by a cytomegalovirus (CMV) promoter. It is 





(See figure on previous page.)

Fig. 8 Effects of cytokine and EtOH treatment on intracellular hHSPB1, MAP2, and GFAP levels in transgenic cultures. a hHSPB1 and MAP2 double immunofluorescence staining in primary neuronal culture from hHSPB1-overexpressing mice $24 \mathrm{~h}$ after EtOH and cytokine treatment. Scale bar: $200 \mu \mathrm{m}$. Red: MAP2; green: hHSPB1; blue: DAPI. b Quantification of MAP2-positive area in transgenic primary neurons. $n=12$. c Quantification of hHSPB1 fluorescent intensity in transgenic primary neurons. $n=12$. $\mathbf{d}$ hHSPB1 and GFAP double immunofluorescence staining in primary astrocyte culture from hHSPB1-overexpressing mice $24 \mathrm{~h}$ after EtOH and cytokine treatment. Scale bar: 50 $\mu \mathrm{m}$. Red: GFAP; green: hHSPB1; blue: DAPI. e Quantification of GFAP fluorescent intensity in transgenic primary astrocytes. $n=9-12$. $\mathbf{f}$ Quantification of hHSPB1 fluorescent intensity in transgenic primary astrocytes. $n=10-11$. Data are expressed as a percentage of untreated cells. Values presented are means \pm SEM. crtl untreated control, EtOH ethanol treatment, cyt cytokine treatment. Statistical analysis: unpaired two-tailed $t$ test. ${ }^{*} p<0.05 ;{ }^{* * *} p<0.001$ compared to control

usually referred to as a constitutively active promoter; however, it has been shown to be upregulated in vitro under certain stress or inflammatory conditions [40-42]. Accordingly, increased transgene expression was observed in response to ethanol treatment in different brain regions of our transgenic mice and in the primary cells as well. Several previous studies have demonstrated that HSPB1 is upregulated upon neurological disorders; therefore, we used this transgenic strain to model the role of HSPB1 in neuroinflammation. Moreover, the expression pattern of hHSPB1 protein in this transgenic model is similar to the one that physiologically occurs in the human brain, as the hHSPB1 protein was found to be synthesized in astrocytes and neurons; however, hHSPB1-positive microglia were rarely detected [43].

Consistent with previous data, our results confirmed that even a single day of ethanol treatment is able to induce high level of apoptosis accompanied by glial cell activation and cytokine production in the developing brain. The presence of hHSPB1 in transgenic animals in itself did not influence the gene expression level of proinflammatory cytokines remarkably. However, hHSPB1 overexpression in combination with ethanol treatment resulted in a significantly higher increase in the level of pro-inflammatory cytokines compared to those observed in ethanol-treated wild-type mice $24 \mathrm{~h}$ after ethanol injection, whereas the level of anti-inflammatory cytokines remained unchanged. These results indicate that hHSPB1 overexpression intensifies the expression of pro-inflammatory cytokines in vivo following acute ethanol treatment.

Pro-inflammatory cytokines are produced in the brain by various cells, such as microglia. At the same time, cytokines affect microglia resulting in their further activation. Upon different immunological stimuli and neuronal injuries, microglia show an immediate response leading to altered morphology and function. These activated microglia cells are implicated in the protection of brain tissue by cleaning cellular debris via phagocytosis, and in inflammatory processes as well [39]. Immunostaining with IBA1 antibody clearly showed that the activation of microglia takes place within $24 \mathrm{~h}$ after a single day of ethanol treatment, as the size of IBA1-positive areas and the proportion of activated microglia cells increased significantly in all brain regions in response to ethanol. Ethanol-treated hHSPB1 mice showed significantly greater IBA1 coverage in certain brain areas than their wild-type littermates, with a nonsignificant trend towards higher proportion of activated microglia. Interestingly, we found differences in the control groups as well, since the thalamus of control transgenic animals showed a significantly higher amount of ramified microglia and lower IBA1 immunoreactivity compared to that of wild-type ones. Our immunohistochemistry data seems to contradict the result of the RTPCR experiments, where we found a higher gene expression of Aif1 in the control transgenic vs wild-type group, while it did not show a further increase in the transgenic animals in response to ethanol treatment. This discrepancy could be derived from the different samplings in the two experiments: in the immunostaining, we analyzed certain brain regions separately, while mRNA was isolated from the whole-brain homogenates for the RT-PCR study. Following brain injury, activated microglia move rapidly towards the damaged neurons leading to a remarkable increase in the local microglial density [44]. Therefore, it could be possible that Aif1 expression is overall invariable in the brain while there are local differences in certain brain regions, similarly what we found in the brain of the transgenic animals at the protein level with and without ethanol treatment. The alterations in the nontreated group could be the result of the fact that HSPB1 is involved in neurodevelopment, contributing to neuronal differentiation and angiogenesis [45]. However, when examining the effect of ethanol treatment, we must acknowledge that activated microglia could play a complex and, in many cases, context-dependent role in the damaged nervous system, as they can contribute to both pathological and reparative processes. Recent studies showed that the depletion of microglia was able to block the expression of ethanol-induced pro-inflammatory factors $[46,47]$, whereas other groups found that the elimination of microglia exacerbated neuronal damage and augmented pro-inflammatory processes after ischemic brain injury [48-50]. In our study, although higher IBA1 immunoreactivity and higher pro-inflammatory cytokine expression were found in ethanol-treated transgenic animals compared to their wild-type littermates, there was no concomitant increase in neuronal loss, suggesting that 
hHSPB1 overexpression may induce diverse or even opposite microglial functions. Therefore, we examined the expression of markers corresponding to M1/M2 microglial polarization and we found that, in response to ethanol treatment, hHSPB1 overexpression enhanced the presence of both microglial phenotypes, since the M2 microglia marker Arg1 and the M1 marker $C d 68$ were also significantly higher in transgenic animals compared to wild-type ones. However, the remarkably high expression of pro-inflammatory cytokines demonstrated that M1 (pro-inflammatory) microglia predominated in hHSPB1 transgenic animals 1 day after ethanol treatment. After ethanol-induced brain injury, microglial recovery could be important in promoting neurogenesis leading to functional recovery [51, 52]. One week after the ethanol treatment, IBA1-stained microglia have recovered their highly ramified processes throughout the brain, accompanied by the decrease of cytokine expression to the normal level. It seems that in the ethanol-treated wild-type animals, microglial recovery could not take place within 1 week, while the level of IBA1 was almost equal in the transgenic groups with or without ethanol treatment.

Astrocytes, the other key cells in neuroinflammation, are special glial cells that have many essential functions in the brain. They respond to different forms of brain injuries through a process called reactive astrogliosis, which has different stages, according to the severity of the injury [53]. The morphological transformation of astrocytes and glial scar formation are partly controlled by pro- and anti-inflammatory cytokines [44]. As ethanol treatment triggered a rapid increase in cytokine expression and microglia activation in our experiment, it is not surprising that Gfap mRNA level was also elevated $24 \mathrm{~h}$ after treatment, showing significantly higher expression in ethanol-treated transgenic animals compared to ethanol-treated wild-type ones. However, no substantial changes were found neither in the morphology of the astrocytes nor in the GFAP coverage on the first day after injection. Instead, a remarkable expansion of GFAPstained cells was observed 1 week later, corresponding with earlier studies showing that the formation of GFAP-positive filaments is a slower process. Moreover, GFAP staining around the blood vessels was also augmented in response to ethanol, especially in hHSPB1overexpressing transgenic brains, leading to a significant difference in the percentage of GFAP-covered area between the two genotypes in certain brain regions. We also studied the inflammatory processes in primary astrocyte cultures in which astrocyte activation was observed, as we detected a pronounced GFAP upregulation within the cells in response to cytokine and ethanol treatments. Astrocytes can have a multitude of functions in the brain; thus, the remarkably high astrocytic
hHSPB1 expression in the present study could potentially modulate these functions and could be key in understanding our observations. HSPB1 has been shown to interact with GFAP and to assist the proper organization of the filament network in astrocytes, which potentially contribute to the maintenance of normal astrocyte function [12]. Furthermore, strong colocalization of HSPB1 with cleaved caspase-3 in reactive astrocytes could promote astroglial survival [54]. HSPB1 may not only have positive effects in glial cells but can also be beneficial for the surrounding damaged tissue [55]. For example, Bechtold and Brown [56] raised the possibility of a synaptic transfer of HSPB1 from glial cells to neurons under stress conditions. In addition, presynaptic localization of HSPB1 in astrocytes could also promote neuronal survival via the restoration of synaptic activity after stress stimuli [56].

We also analyzed which cell type of the brain tissue could be responsible for the highly elevated levels of pro-inflammatory cytokines in hHSPB1 transgenic mice. Our in vitro results demonstrated that microglia are the primary TNF $\alpha$-producing cell type under inflammatory conditions. In terms of astrocytes, both wild-type and transgenic cells had a basal TNF $\alpha$ production, which increased after cytokine treatment in the wild-type culture, but not in the transgenic one. Considering that HSPB1 was previously shown to interact with Annexin A1 and thereby lower the release of TNF $\alpha$ in reactive astrocytes $[17,57]$, it is possible that the greatly elevated level of intracellular hHSPB1 in astrocytes may directly affect their TNFa production. The observation that ethanol treatment did not induce TNF $\alpha$ release in glial cultures may be explained by the fact that the inflammationinducing effect of ethanol is partly related to neuronal damage. For example, in the brain of adult rodents, which are less sensitive to the harmful effects of ethanol compared to fetuses, only a low degree of neurodegeneration or decline in neurogenesis was observed and, in parallel, no significant changes in protein levels of proinflammatory cytokines were detected $[22,58]$. On the other hand, the effects of ethanol are potentiated by its metabolites: acetaldehyde had a greater impact on astrocyte and oligodendrocyte cultures than ethanol $[59,60]$. Overall, these findings are in accordance with previous studies, in which activated microglia were identified as the main source of pro-inflammatory cytokines, whereas TNF $\alpha$ production by astrocytes was lower even after activating stimuli $[61,62]$. We consider the use of serum for the culture of our primary cells as a limitation of our study because of potential priming effects. We should note that based on our present results as well as literature data, primary astro- and microglia cells despite being exposed to serum showed low expression of inflammatory mediators and activation markers [63, 64]. 
It is very interesting that, after cytokine treatment, microglia from hHSPB1 transgenic animals were seemingly in a further increased inflammatory status by secreting a higher amount of TNFa than wild-type cells, although microglia did not express appreciable amount of the transgene. This finding suggests a potential indirect effect of hHSPB1 on microglia activation. Based on previous data, not only the initial microglia response can affect the subsequent astrocyte activation, but microglial function could also depend on the crosstalk with astrocytes. For example, NFkB-mediated astrocytic expression of WNT induces microglia proliferation or the ATP released from astrocyte can act on the purinergic receptors of microglia with concomitant induction of $\mathrm{Ca}^{2+}$ signaling and IL-1 $\beta$ containing vesicula formation [65-68]. Astrocytes can also mediate microglial function and distribution by secreting a plethora of inflammatory cytokines and chemokines or through direct cell-cell interactions [69-71]. Therefore, we hypothesize that elevated intracellular hHSPB1 levels in astrocytes remaining in microglial cultures could have an effect on the release of these factors, contributing to the increased microglial activation. HSPB1 has been demonstrated to regulate several signaling cascades, such as inflammationassociated NFKB or p38 mitogen-activated protein kinase (MAPK) pathways; therefore, it could modulate the expression of numerous factors. Intracellular HSPB1 was also shown to induce the transcription of inflammatory mediators by participating in the stabilization of their mRNA [72]. In addition, astrocyte cell culture treated with HSPB1 exhibited an increased level of IL- 8 and decreased production of TGF- $\beta 1$ and CD40 ligand [16]. However, based on these findings, it would be of interest to fully elucidate the activation circuit between astrocytes and microglia in hHSPB1-overexpressing mice.

These results clearly demonstrate that the overexpression of hHSPB1 protein promotes glial activation and cytokine response upon acute ethanol treatment. Despite that HSPB1 was previously seen as an anti-inflammatory factor, our observation is in accordance with a growing number of evidence suggesting a more complicated, context-dependent role for this protein in inflammation [73]. An earlier report using HeLa cells showed that HSPB1 is associated with the expression of proinflammatory factors and involved in the IL-1 and TNFinduced signaling [72]. In C6 glioma cells, the chaperone activity of HSPB1 contributed to the production of the pro-inflammatory cytokine, IL-6, whereas $\mathrm{T}$ cells from $\mathrm{HSPB}^{-1-}$ mice exhibited reduced secretion of TNF and IL-2 [74, 75]. Recombinant HSPB1 treatment also resulted in enhanced pro-inflammatory cytokine expression in vitro $[18,76,77]$. Although the majority of the literature describes extracellular heat-shock proteins as regulators of inflammation, we could not detect hHSPB1 in the supernates of the primary cell cultures in our experiments. Thus, it is reasonable to suspect that the intracellular form of the protein is the major source of inflammation modulation in this scenario.

In order to learn more about the overall outcome of the amplified pro-inflammatory cytokine production in hHSPB1-overexpressing mice, ethanol-induced cell death was studied using TUNEL assay. In the brain of the control, saline-treated animals, the low extent of apoptosis could correspond to the physiological, programmed cell death occurring in the developing brain [21]. However, even a single day of ethanol treatment dramatically increased the number of apoptotic cells in most of the studied brain areas. The observed differences between the brain regions were corresponding to their previously described susceptibility to alcoholinduced cell death and to their described sensitivity to alcohol in different developmental stages [21]. Intriguingly, despite the remarkably high level of $\operatorname{Tnf}$, we found a slightly lower amount of TUNEL-positive apoptotic cells in the brains of hHSPB1 animals compared with those of wild-types after ethanol treatment. TNF $\alpha$ is a described inducer of apoptosis in the nervous system acting in a dose-dependent manner [78-80]. On the other hand, numerous in vivo and in vitro studies proved that HSPB1 contributes to the prevention of neuronal cell death [81-84]. Previously, we demonstrated the protective effects of hHSPB1 on neurons after both acute and chronic ethanol treatment in adult mice [37]. The neuroprotective effect of the enhanced intracellular hHSPB1 could partly be based on its ability to interact with neurofilaments and with the actin network, and by this supporting the structural integrity of neurons $[85,86]$. HSPB1 can also affect the TNF $\alpha-$ induced cell death. HSPB1 prevented TNF $\alpha$-mediated apoptosis by modulating cellular glutathione levels [87], by mediating the activity of NFkB $[88,89]$, or the p38/ extracellular signal-regulated kinase (ERK) signaling pathways [90]. Furthermore, HSPB1 was shown to prevent caspase-mediated apoptosis, which can also be initiated by TNF $\alpha$ [91, 92]. Based on these observations, we assume that the elevated level of hHSPB1 could compensate the apoptosis-inducing effect of the highly increased $\operatorname{Tnf}$ in the hHSBP1-overexpressing mice. Moreover, it is worth mentioning that elevated levels of pro-inflammatory cytokines in the nervous system can also have beneficial effects. For example, depending on the target receptor it acts on, TNF $\alpha$ can be involved in demyelination as well as in neuronal regeneration, whereas neurotrophic factors were found to be upregulated after acute IL-1 $\beta$ administration [93, 94]. Furthermore, it has been recently discovered that chronic IL-1 $\beta$ and TNF $\alpha$ treatment activates an astrocyte phenotype that supports neuronal viability [95]. 


\section{Conclusions}

Taken together, our work provides in vivo evidence that hHSPB1 overexpression is associated with enhanced pro-inflammatory response in a mouse model of ethanol-induced acute neuroinflammation. Notably, hHSPB1 overexpression modulated the inflammatory processes evoked by ethanol resulting in higher expression of pro-inflammatory cytokines and increased glial cell activation. The findings presented here could shed light on an unknown balancing role of hHSPB1 in inflammation regulation as the increased inflammation in the hHSPB1-overexpressing mice was not accompanied by increased neuronal damage. Thus, the data presented by this study could help to understand the potential regulatory role of hHSPB1 in neuroinflammation.

\section{Supplementary Information}

The online version contains supplementary material available at https://doi. org/10.1186/s12974-020-02070-2.

Additional file 1. Supplementary materials

\begin{abstract}
Abbreviations
Aif1: Allograft inflammatory factor 1; Arg1: Arginase 1; BSA: Bovine serum albumin; CD40: Cluster of differentiation 40; Cd68: Cluster of differentiation 68; DAB: 3,3'-Diaminobenzidine; DAPI: 4',6-Diamidino-2-phenylindole; ELISA: Enzyme-linked immunosorbent assay; ERK: Extracellular signalregulated kinase; FITC: Fluorescein isothiocyanate; Gapdh: Glyceraldehyde 3phosphate dehydrogenase; GFAP: Glial fibrillary acidic protein; HSP: Heatshock protein; HSPB1: Heat-shock protein B1; IBA1: Ionized calcium-binding adaptor molecule 1; IL: Interleukin; IKBa: Inhibitor of nuclear factor kappa B-a iNos: Inducible nitric oxide synthase; MAP 2: Microtubule-associated protein2; MAPK: Mitogen-activated protein kinase; Mrc1: Mannose receptor C-type 1; NEUN: Neuronal nuclear antigen; NFkB: Nuclear factor kappa B; OCT compound: Optimal cutting temperature compound; PBS: Phosphatebuffered saline; PBST: Phosphate-buffered saline with Tween 20; PFA: Paraformaldehyde; RIPA: Radioimmunoprecipitation assay; SDS: Sodium dodecyl sulfate; TGF- $\beta 1$ : Transforming growth factor- $\beta 1$; TNFa: Tumor necrosis factor-a; TUNEL: Terminal deoxynucleotidyl transferase dUTP nick end labeling; Vim: Vimentin
\end{abstract}

\section{Acknowledgements}

The authors thank Ibolya Kis-Sebestyén for her technical assistance.

\section{Authors' contributions}

MET, MS, MAD, BD, and FRW conceived and designed the experiments. BD, MET, FRW, JPV, BB, PH, EM, and ZH performed the experiments. BD, MET, FRW, JPV, BB, PH, and AK analyzed and interpreted the data. TB and $\mathrm{PH}$ performed the deep learning experiment. TS, EB, BC, PH, LF, BP, MS, and LV helped to conduct the experiments, provided guidance and assistance in data analysis, and contributed reagents/materials/analysis tools. BD, MET, and FRW wrote the original draft. MET, MS, and MAD revised and edited the manuscript. All authors read and approved the final manuscript.

\section{Funding}

This study was funded by the National Research, Development and Innovation Office, Hungary (GINOP 2.3.2.15.2016-00060, GINOP 2.3.2.-15.2016-00040) and by the Hungarian Scientific Research Fund (OTKA NN-111006). F.R.W. was supported by the National Research, Development and Innovation Office, Hungary (OTKA PD-128480), by the János Bolyai Research Fellowship of the Hungarian Academy of Sciences, and by the New National Excellence Program Bolyai+ fellowship (UNKP-19-4-SZTE-42; UNKP-20-5-SZTE-672) of the Ministry for Innovation and Technology, Hungary.

\section{Availability of data and materials}

The datasets used and/or analyzed during the current study are available from the last authors on reasonable request.

\section{Ethics approval and consent to participate}

The study was approved by the regional National Food Chain Safety Agency and Animal Health Directorate (Csongrád-county, Hungary).

\section{Consent for publication}

Not applicable.

\section{Competing interests}

The authors declare that they have no competing interests.

\section{Author details}

${ }^{1}$ Institute of Biochemistry, Biological Research Centre, Temesvári krt. 62, Szeged H-6726, Hungary. ${ }^{2}$ Doctoral School in Biology, University of Szeged, Szeged, Hungary. ${ }^{3}$ Institute of Biophysics, Biological Research Centre, Szeged, Hungary. ${ }^{4}$ Doctoral School in Theoretical Medicine, University of Szeged, Szeged, Hungary. ${ }^{5}$ Doctoral School of Informatics, ELTE Eötvös Loránd University, Budapest, Hungary. ${ }^{6}$ Department of Medical Chemistry, Faculty of Medicine, University of Szeged, Szeged, Hungary. ${ }^{7}$ Institute of Medical Biology, University of Troms $\varnothing$, Troms $\varnothing$, Norway. ${ }^{8}$ Institute for Molecular Medicine Finland (FIMM), University of Helsinki, Helsinki, Finland.

Received: 7 August 2020 Accepted: 30 December 2020

Published online: 10 January 2021

\section{References}

1. Banjara M, Ghosh C. Sterile neuroinflammation and strategies for therapeutic intervention. Int J Inflam. 2017;2017:1-20.

2. Dukay B, Csoboz B, Tóth ME. Heat-shock proteins in neuroinflammation. Front Pharmacol. 2019;10:920.

3. Nakamoto H, Vígh L. The small heat shock proteins and their clients. Cell Mol Life Sci. 2007;64:294-306.

4. Schwarz L, Vollmer G, Richter-Landsberg C. The small heat shock protein HSP25/27 (HspB1) is abundant in cultured astrocytes and associated with astrocytic pathology in progressive supranuclear palsy and corticobasal degeneration. Int J Cell Biol. 2010;717520:10.

5. Rioux DA, Murphy K, Esser MJ, Currie RW. Neurodegenerative diseases, sex differences and the $27 \mathrm{kDa}$ heat shock protein in the nervous system. In: The big book on small heat shock proteins. Cham: Springer; 2015. p. 335-63.

6. Imura T, Shimohama S, Sato M, Nishikawa H, Madono K, Akaike A, et al. Differential expression of small heat shock proteins in reactive astrocytes after focal ischemia: possible role of beta-adrenergic receptor. J Neurosci. 1999:19:9768-79.

7. Renkawek K, Bosman GICGM, de Jong WW. Expression of small heat-shock protein hsp 27 in reactive gliosis in Alzheimer disease and other types of dementia. Acta Neuropathol. 1994;87:511-9.

8. Wilhelmus M, Otte-Höller I, Wesseling P, de Waal RMW, Boelens WC, Verbeek MM. Specific association of small heat shock proteins with the pathological hallmarks of Alzheimer's disease brains. Neuropathol Appl Neurobiol. 2006;32:119-30.

9. Sharp FR, Zhan X, Liu DZ. Heat shock proteins in the brain: role of Hsp70, Hsp 27, and HO-1 (Hsp32) and their therapeutic potential. Transl Stroke Res. 2013;4:685-92.

10. Bartelt-Kirbach B, Slowik A, Beyer C, Golenhofen N. Upregulation and phosphorylation of HspB1/Hsp25 and HspB5/aB-crystallin after transient middle cerebral artery occlusion in rats. Cell Stress Chaperones. 2017;22: 653-63.

11. Bechtold DA, Brown IR. Induction of Hsp27 and Hsp32 stress proteins and vimentin in glial cells of the rat hippocampus following hyperthermia. Neurochem Res. 2003:28:1163-73.

12. Der Perng M, Cairns L, Van Den IJssel P, Prescott A, Hutcheson AM, Quinlan RA. Intermediate filament interactions can be altered by HSP27 and aBcrystallin. J Cell Sci. 1999;112:2099-112.

13. Nefedova W, Sudnitsyna MV, Gusev NB. Interaction of small heat shock proteins with light component of neurofilaments (NFL). Cell Stress Chaperones. 2017;22:467-79.

14. Stetler RA, Gao Y, Signore AP, Cao G, Chen J. HSP27: mechanisms of cellular protection against neuronal injury. Curr Mol Med. 2009;9:863-72. 
15. Giuliano JS, Lahni PM, Wong HR, Wheeler D. Pediatric sepsis—part V: extracellular heate shock proteins: alarmins for the host immune system. PubMed - NCBI. Open Inflamm J. 2011;4:49-60.

16. Bruinsma IB, de Jager M, Carrano A, Versleijen AAM, Veerhuis R, Boelens W, et al. Small heat shock proteins induce a cerebral inflammatory reaction. J Neurosci. 2011;31:11992-2000.

17. Liu L, An D, Xu J, Shao B, Li X, Shi J. Ac2-26 induces IKK $\beta$ degradation through chaperone-mediated autophagy via HSPB1 in NCM-treated microglia. Front Mol Neurosci. 2018;11. https://doi.org/10.3389/fnmol.2018.00076.

18. Salari S, Seibert T, Chen Y-X, Hu T, Shi C, Zhao X, et al. Extracellular HSP27 acts as a signaling molecule to activate NF-KB in macrophages. Cell Stress Chaperones. 2013;18:53-63.

19. Shi Y, Jiang $X$, Zhang L, Pu H, Hu X, Zhang W, et al. Endothelium-targeted overexpression of heat shock protein 27 ameliorates blood-brain barrier disruption after ischemic brain injury. Proc Natl Acad Sci U S A. 2017;114: E1243-52

20. Tóth ME, Szegedi V, Varga E, Juhász G, Horváth J, Borbély E, et al. Overexpression of Hsp27 ameliorates symptoms of Alzheimer's disease in APP/PS1 mice. Cell Stress Chaperones. 2013;18:759-71.

21. Ikonomidou C, Bittigau P, Koch C, Genz K, Stefovska V, Hörster F. Ethanolinduced apoptotic neurodegeneration and fetal alcohol syndrome. Science (80- ) 2000; 287: 1056-1060.

22. Saito M, Chakraborty G, Hui M, Masiello K, Saito M. Ethanol-induced neurodegeneration and glial activation in the developing Brain. Brain Sci. 2016;6. https://doi.org/10.3390/brainsci6030031.

23. Ayoub AE, Salm AK. Increased morphological diversity of microglia in the activated hypothalamic supraoptic nucleus. J Neurosci. 2003;23:7759-66.

24. Doyle HH, Eidson LN, Sinkiewicz DM, Murphy AZ. Sex differences in microglia activity within the periaqueductal gray of the rat: a potential mechanism driving the dimorphic effects of morphine. J Neurosci. 2017:37:3202-14.

25. Hollandi R, Diósdi Á, Hollandi G, Moshkov N, Horváth P. AnnotatorJ: an ImageJ plugin to ease hand-annotation of cellular compartments. Mol Bio Cell. 2020;31:2179-86.

26. Paszke A, Gross S, Chintala S, Chanan G, Yang E, DeVito Z, et al. Automatic differentiation in pytorch. 2017; Preprint. https://openreview.net/pdf?id= BJJsrmfCZ.

27. Redmon J, Farhadi A. YOLOv3: an incremental improvement. 2018; arXiv 1804.02767 Cs.

28. Wang C-Y, Liao H-YM, Yeh I-H, Wu Y-H, Chen P-Y, Hsieh J-W. CSPNet: a new backbone that can enhance learning capability of CNN. 2019; http://arxiv. org/abs/1911.11929.

29. Kingma D, Ba J. Adam: a method for stochastic optimization. In: Proceedings of the 3rd International Conference on Learning Representations: 2015; https://iclr.cc/archive/www/doku.php\%3Fid=iclr2015: accepted-main.html.

30. Pacifici M, Peruzzi F. Isolation and culture of rat embryonic neural cells: A quick protocol. J Vis Exp. 2012;63:e3965.

31. Lénárt N, Walter FR, Bocsik A, Sántha P, Tóth ME, Harazin A, et al. Cultured celels of the blood-brain barrier from apolipoprotein B-100 transgenic mice: effects of oxidized low-density lipoprotein treatment. Fluids Barriers CNS 2015;12:17.

32. Lian $\mathrm{H}$, Roy $\mathrm{E}$, Zheng $\mathrm{H}$. Protocol for primary microglial culture preparation BIO-PROTOCOL 2016;6. https://doi.org/10.21769/bioprotoc.1989.

33. Haorah J, Ramirez SH, Floreani N, Gorantla S, Morsey B, Persidsky Y. Mechanism of alcohol-induced oxidative stress and neuronal injury. Free Radic Biol Med. 2008;45:1542-50.

34. Chapp AD, Behnke JE, Driscoll KM, Fan Y, Hoban E, Shan Z, et al. Acetate mediates alcohol excitotoxicity in dopaminergic-like PC12 cells. ACS Chem Neurosci. 2019;10:235-45.

35. Haorah J, Knipe B, Gorantla S, Zheng J, Persidsky Y. Alcohol-induced bloodbrain barrier dysfunction is mediated via inositol 1,4,5-triphosphate receptor (IP3R)-gated intracellular calcium release. J Neurochem. 2007;100:324-36.

36. Harazin A, Bocsik A, Barna L, Kincses A, Váradi J, Fenyvesi F, et al. Protection of cultured brain endothelial cells from cytokine-induced damage by amelanocyte stimulating hormone. PeerJ. 2018;2018:e4774.

37. Tóth ME, Gonda S, Vigh L, Santha M. Neuroprotective effect of small heat shock protein, Hsp27, after acute and chronic alcohol administration. Cell Stress Chaperones. 2010;15:807-17.

38. Brownell SE, Becker RA, Steinman L. The protective and therapeutic function of small heat shock proteins in neurological diseases. Front Immunol. 2012; 3. https://doi.org/10.3389/fimmu.2012.00074.
39. Yang JY, Xue $X$, Tian $H$, Wang XX, Dong YX, Wang F, et al. Role of microglia in ethanol-induced neurodegenerative disease: pathological and behavioral dysfunction at different developmental stages. Pharmacol Ther. 2014;144:321-37.

40. Prösch S, Staak K, Stein J, Liebenthal C, Stamminger T, Volk HD, et al. Stimulation of the human cytomegalovirus IE enhancer/promoter in HL-60 cells by TNFa is mediated via induction of NF-KB. Virology. 1995;208:197-206.

41. Bruening W, Giasson B, Mushynski W, Durham H. Activation of stress-activated MAP protein kinases up-regulates expression of transgenes driven by the cytomegalovirus immediate/early promoter. Nucleic Acids Res. 1998;26:486-9.

42. Svensson RU, Barnes JM, Rokhlin OW, Cohen MB, Henry MD. Chemotherapeutic agents up-regulate the cytomegalovirus promoter: implications for bioluminescence imaging of tumor response to therapy. Cancer Res. 2007:67:10445-54.

43. Satoh J-I, Kim SU. Differential expression of heat shock protein HSP27 in human neurons and glial cells in culture. J Neurosci Res. 1995:41:805-18.

44. Raivich G, Bohatschek M, Kloss CUA, Werner A, Jones LL, Kreutzberg GW. Neuroglial activation repertoire in the injured brain: graded response, molecular mechanisms and cues to physiological function. Brain Res Rev. 1999;30:77-105.

45. Miller DJ, Fort PE. Heat shock proteins regulatory role in neurodevelopment. Front Neurosci. 2018;12:821.

46. Coleman LG, Zou J, Crews FT. Microglial depletion and repopulation in brain slice culture normalizes sensitized proinflammatory signaling. Neuroinflammation. 2020;17:27.

47. Walter TJ, Crews FT. Microglial depletion alters the brain neuroimmune response to acute binge ethanol withdrawal. J Neuroinflammation. 2017;14. https://doi.org/10.1186/s12974-017-0856-Z

48. Szalay G, Martinecz B, Lénárt N, Környei Z, Orsolits B, Judák L, et al. Microglia protect against brain injury and their selective elimination dysregulates neuronal network activity after stroke. Nat Commun. 2016;7:11499.

49. Tsuji S, Di Martino E, Mukai T, Tsuji S, Murakami T, Harris RA, et al. Aggravated brain injury after neonatal hypoxic ischemia in microgliadepleted mice. J Neuroinflammation. 2020;17:111.

50. Jin W-N, Shi SX-Y, Li Z, Li M, Wood K, Gonzales RJ, et al. Depletion of microglia exacerbates postischemic inflammation and brain injury. J Cereb Blood Flow Metab. 2017:37:2224-36.

51. Zhao YN, Wang F, Fan YX, Ping GF, Yang JY, Wu CF. Activated microglia are implicated in cognitive deficits, neuronal death, and successful recovery following intermittent ethanol exposure. Behav Brain Res. 2013;236:270-82.

52. McClain JA, Morris SA, Deeny MA, Marshall SA, Hayes DM, Kiser ZM, Nixon K. Adolescent binge alcohol exposure induces long-lasting partial activation of microglia. - PubMed - NCBI. Brain Behav Immun. 2011;25:S120-8.

53. Sofroniew MV, Vinters HV. Astrocytes: biology and pathology. Acta Neuropathol. 2010;119:7-35.

54. Villapol S, Acarin L, Faiz M, Castellano B, Gonzalez B. Survivin and heat shock protein 25/27 colocalize with cleaved caspase-3 in surviving reactive astrocytes following excitotoxicity to the immature brain. Neuroscience. 2008;153:108-19.

55. Acarin L, Paris J, González B, Castellano B. Glial expression of small heat shock proteins following an excitotoxic lesion in the immature rat brain. Glia. 2002;38:1-14.

56. Bechtold DA, Brown IR. Heat shock proteins Hsp27 and Hsp32 localize to synaptic sites in the rat cerebellum following hyperthermia. Brain Res Mol Brain Res. 2000;75:309-20.

57. Shijo M, Hamasaki H, Honda H, Suzuki S, Tachibana M, Ago T, et al. Upregulation of Annexin A1 in reactive astrocytes and its subtle induction in microglia at the boundaries of human brain infarcts. J Neuropathol Exp Neurol. 2019;78. https://doi.org/10.1093/JNEN/NLZ079.

58. Qin L, He J, Hanes RN, Pluzarev O, Hong JS, Crews FT. Increased systemic and brain cytokine production and neuroinflammation by endotoxin following ethanol treatment. J Neuroinflammation. 2008;5:10.

59. Signorini-Allibe N, Gonthier B, Lamarche F, Eysseric H, Barret L. Chronic consumption of ethanol leads to substantial cell damage in cultured rat astrocytes in conditions promoting acetaldehyde accumulation. Alcohol Alcohol. 2005:40:163-71

60. Coutts DJC, Harrison NL. Acetaldehyde, not ethanol, impairs myelin formation and viability in primary mouse oligodendrocytes. Alcohol Clin Exp Res. 2015:39:455-62.

61. Welser-Alves JV, Milner R. Microglia are the major source of TNF-a and TGF$\beta 1$ in postnatal glial cultures; regulation by cytokines, lipopolysaccharide, and vitronectin. Neurochem Int. 2013;63:47-53. 
62. Lambertsen KL, Meldgaard M, Ladeby R, Finsen B. A quantitative study of microglial-macrophage synthesis of tumor necrosis factor during acute and late focal cerebral ischemia in mice. J Cereb Blood Flow Metab. 2005;25:119-35.

63. Lively $\mathrm{S}$, Lam D, Wong R, Schlichter LC. Comparing effects of transforming growth factor $\beta 1$ on microglia from rat and mouse: transcriptional profiles and potassium channels. Front Cell Neurosci. 2018:12:115.

64. Lively S, Schlichter LC. The microglial activation state regulates migration and roles of matrix-dissolving enzymes for invasion. J Neuroinflammation. 2013;10:843.

65. Ouali Alami N, Schurr C, Olde Heuvel F, Tang L, Li Q, Tasdogan A, et al. NF$\mathrm{KB}$ activation in astrocytes drives a stage-specific beneficial neuroimmunological response in ALS. EMBO J. 2018;37. https://doi.org/10. 15252/embj.201798697.

66. Verderio C, Matteoli M. ATP mediates calcium signaling between astrocytes and microglial cells: modulation by IFN-ү. J Immunol. 2001;166:6383-91.

67. Davalos D, Grutzendler J, Yang G, Kim JV, Zuo Y, Jung S, et al. ATP mediates rapid microglial response to local brain injury in vivo. Nat Neurosci. 2005;8: 752-8.

68. Bianco F, Pravettoni E, Colombo A, Schenk U, Möller T, Matteoli M, et al. Astrocyte-derived ATP induces vesicle shedding and $\mathrm{IL}-1 \beta$ release from microglia. J Immunol. 2005;174:7268-77.

69. He M, Dong H, Huang Y, Lu S, Zhang S, Qian Y, et al. Astrocyte-derived CCL2 is associated with M1 activation and recruitment of cultured microglial cells. Cell Physiol Biochem. 2016;38:859-70.

70. Farina C, Aloisi F, Meinl E. Astrocytes are active players in cerebral innate immunity. Trends Immunol. 2007;28:138-45

71. Lana D, Ugolini F, Wenk GL, Giovannini MG, Zecchi-Orlandini S, Nosi D. Microglial distribution, branching, and clearance activity in aged rat hippocampus are affected by astrocyte meshwork integrity: evidence of a novel cell-cell interglial interaction. FASEB J. 2019;33:4007-20.

72. Alford KA, Glennie S, Turrell BR, Rawlinson L, Saklatvala J, Dean JLE. Heat shock protein 27 functions in inflammatory gene expression and transforming growth factor- $\beta$-activated kinase-1 (TAK1)-mediated signaling. J Biol Chem. 2007;282:6232-41.

73. Van Noort JM, Bsibsi M, Nacken P, Gerritsen WH, Amor S. The link between small heat shock proteins and the immune system. Int J Biochem Cell Biol. 2012:44:1670-9.

74. Tanabe K, Matsushima-Nishiwaki R, Dohi S, Kozawa O. Phosphorylation status of heat shock protein 27 regulates the interleukin-1 $\beta$-induced interleukin-6 synthesis in C6 glioma cells. Neuroscience. 2010;170:1028-34.

75. Breed ER, Hilliard CA, Yoseph B, Mittal R, Liang Z, Chen CW, et al. The smal heat shock protein HSPB1 protects mice from sepsis. Sci Rep. 2018:8:1-10.

76. Jin C, Cleveland JC, Ao L, Li J, Zeng Q, Fullerton DA, et al. Human myocardium releases heat shock protein 27 (HSP27) after global ischemia: the proinflammatory effect of extracellular HSP27 through toll-like receptor (TLR)-2 and TLR4. Mol Med. 2014;20:280-9.

77. Yusuf N, Nasti TH, Huang C-M, Huber BS, Jaleel T, Lin H-Y, et al. Heat shock proteins HSP27 and HSP70 are present in the skin and are important mediators of allergic contact hypersensitivity. J Immunol. 2009;182:675-83.

78. Rath PC, Aggarwal BB. TNF-induced signaling in apoptosis. J Clin Immunol. 1999;19:350-64.

79. Talley AK, Dewhurst S, Perry SW, Dollard SC, Gummuluru S, Fine SM, et al. Tumor necrosis factor alpha-induced apoptosis in human neuronal cells: protection by the antioxidant $\mathrm{N}$-acetylcysteine and the genes bcl-2 and crmA. Mol Cell Biol. 1995:15:2359-66.

80. Chen N, Wei F, Wang L, Cui S, Wan Y, Liu S. Tumor necrosis factor alpha induces neural stem cell apoptosis through activating p38 MAPK pathway. Neurochem Res. 2016;41:3052-62.

81. Wagstaff MJD, Collaço-Moraes Y, Smith J, De Belleroche JS, Coffin RS, Latchman DS. Protection of neuronal cells from apoptosis by Hsp27 delivered with a herpes simplex virus-based vector. J Biol Chem. 1999;274: 5061-9.

82. Van Der Weerd L, Taeriq Akbar M, Aron Badin R, Valentim LM, Thomas DL, Wells DJ, et al. Overexpression of heat shock protein 27 reduces cortical damage after cerebral ischemia. J Cereb Blood Flow Metab. 2010;30:849-56.

83. Akbar MT, Lundberg AMC, Liu K, Vidyadaran S, Wells KE, Dolatshad H, et al. The neuroeprotective effects of heat shock protein 27 overexpression in transgenic animals against kainate-induced seizures and hippocampal cell death. J Biol Chem. 2003;278:19956-65.

84. Penke B, Bogár F, Crul T, Sántha M, Tóth ME, Vígh L. Heat shock proteins and autophagy pathways in neuroprotection: from molecular bases to pharmacological interventions. Int J Mol Sci. 2018;19. https://doi.org/10. 3390/ijms19010325.

85. Zhai J, Lin H, Julien J-P, Schlaepfer WW. Disruption of neurofilament network with aggregation of light neurofilament protein: a common pathway leading to motor neuron degeneration due to Charcot-MarieTooth disease-linked mutations in NFL and HSPB1. Hum Mol Genet. 2007;16: 3103-16.

86. Williams KL, Rahimtula M, Mearow KM. Hsp27 and axonal growth in adult sensory neurons in vitro. BMC Neurosci. 2005;6:24.

87. Mehlen P, Kretz-Remy C, Préville X, Arrigo AP. Human hsp27, Drosophila hsp27 and human alphaB-crystallin expression-mediated increase in glutathione is essential for the protective activity of these proteins against TNFalpha-induced cell death. EMBO J. 1996;15:2695-706.

88. Parcellier A, Schmitt E, Gurbuxani S, Seigneurin-Berny D, Pance A, Chantôme A, et al. HSP27 is a ubiquitin-binding protein involved in I-kBa proteasomal degradation. Mol Cell Biol. 2003;23:5790-802.

89. Bin ZZ, Xiang HG, Jia $\sqcup$, Ma J, Jun $Y Q$, Cao $Y$, et al. Up-regulation of heat shock protein 27 inhibits apoptosis in lumbosacral nerve root avulsioninduced neurons. Sci Rep. 2019;9:1-10.

90. Qi Z, Shen L, Zhou H, Jiang Y, Lan L, Luo L, et al. Phosphorylation of heat shock protein 27 antagonizes TNF-a induced HeLa cell apoptosis via regulating TAK1 ubiquitination and activation of p38 and ERK signaling. Cell Signal. 2014;26:1616-25.

91. Gordon R, Anantharam V, Kanthasamy AG, Kanthasamy A. Proteolytic activation of proapoptotic kinase protein kinase $C \delta$ by tumor necrosis factor a death receptor signaling in dopaminergic neurons during neuroinflammation. J Neuroinflammation. 2012;9:82.

92. Bruey JM, Ducasse C, Bonniaud P, Ravagnan L, Susin SA, Diaz-Latoud C, et al. Hsp27 negatively regulates cell death by interacting with cytochrome c. Nat Cell Biol. 2000;2:645-52.

93. Arnett HA, Mason J, Marino M, Suzuki K, Matsushima GK, Ting JPY. TNFa promotes proliferation of oligodendrocyte progenitors and remyelination. Nat Neurosci. 2001:4:1116-22.

94. Song C, Zhang Y, Dong Y. Acute and subacute IL-1 $\beta$ administrations differentially modulate neuroimmune and neurotrophic systems: possible implications for neuroprotection and neurodegeneration. J Neuroinflammation. 2013:10:59.

95. Hyvärinen T, Hagman S, Ristola M, Sukki L, Veijula K, Kreutzer J, et al. Costimulation with $\mathrm{IL}-1 \beta$ and TNF- $\alpha$ induces an inflammatory reactive astrocyte phenotype with neurosupportive characteristics in a human pluripotent stem cell model system. Sci Rep. 2019:9:16944.

\section{Publisher's Note}

Springer Nature remains neutral with regard to jurisdictional claims in published maps and institutional affiliations.

Ready to submit your research? Choose BMC and benefit from:

- fast, convenient online submission

- thorough peer review by experienced researchers in your field

- rapid publication on acceptance

- support for research data, including large and complex data types

- gold Open Access which fosters wider collaboration and increased citations

- maximum visibility for your research: over $100 \mathrm{M}$ website views per year

At BMC, research is always in progress.

Learn more biomedcentral.com/submission 